\title{
Implementation of One and Two-Step Generalized Integral Representation Methods (GIRMs)
}

\author{
Hideyuki Niizato ${ }^{1}$, Gantulga Tsedendorj ${ }^{2}$, Hiroshi Isshiki $^{3}$ \\ ${ }^{1}$ Hitachi Zosen Corporation, Osaka, Japan \\ ${ }^{2}$ Department of Mathematics, National University of Mongolia, Ulaanbaatar, Mongolia \\ ${ }^{3}$ IMA, Institute of Mathematical Analysis, Osaka, Japan \\ Email address: \\ niizato@hitachizosen.co.jp (H. Niizato), gantulga@smcs.num.edu.mn (G. Tsedendorj), isshiki@dab.hi-ho.ne.jp (H. Isshiki)
}

\section{To cite this article:}

Hideyuki Niizato, Gantulga Tsedendorj, Hiroshi Isshiki. Implementation of One and Two-Step Generalized Integral Representation Methods (GIRMs). Applied and Computational Mathematics. Special Issue: Integral Representation Method and its Generalization.

Vol. 4, No. 3-1, 2015, pp. 59-77. doi: 10.11648/j.acm.s.2015040301.15

\begin{abstract}
In this study, we summarize and implement one- and two-step Generalized Integral Representation Methods (GIRMs). Although GIRM requires matrix inversion, the solution is stable and the accuracy is high. Moreover, it can be applied to an irregular mesh. In order to validate the theory, we apply one- and two-step GIRMs to the one-dimensional Initial and Boundary Value Problem for advective diffusion. The numerical experiments are conducted and the approximate solutions coincide with the exact ones in both cases. The corresponding computer codes implemented in most popular computational languages are also given.
\end{abstract}

Keywords: Initial and Boundary Value Problem (IBVP), Generalized Fundamental Solution,

Generalized Integral Representation Method (GIRM), Implementation of GIRM, Computer Codes

\section{Introduction}

Integral Representation Method (IRM) and its generalization - Generalized Integral Representation Method (GIRM) are one of the most convenient methods to numerically solve Initial and Boundary Value Problems (IBVP) such as advection-diffusion type equations. Both methods can be applied to an irregular mesh, and the solution is stable and accurate. In Ref. [1], generalization from IRM to GIRM is discussed not only from the theoretical viewpoint, but also from the computational aspects. Comparison and relationship with other numerical methods are also given. As an example, GIRM is applied to one- and two-dimensional diffusion problems and two-dimensional Burgers' equation. Moreover, it is also shown that the determination of a fundamental solution for GIRM is always possible in advance.

As another demonstration, GIRM is applied to fluid dynamic motion of gas or particles to obtain the accurate numerical solutions [2]. The numerical results by the GIRM are compared with the solutions by Finite Difference Method (FDM). The GIRM produces reasonable and accurate numerical solutions.
In the present study, a brief summary of the theory of Oneand Two-step Generalized Integral Representation Methods (GIRMs) are presented. To validate the theory, numerical experiments of the one-dimensional IBVP for advective diffusion are conducted. Highly accurate numerical results are obtained in both cases of One- and Two-step GIRMs in admissible time periods. The corresponding computer codes implemented in widely used programming languages such as Matlab, $\mathrm{C}$ and FORTRAN are also given.

\section{One-Step Generalized Integral Representation Method (1-Step GIRM)}

\subsection{Summary of Theory}

We discuss numerical solutions of the IBVP for advective diffusion. For simplicity, we apply 1-step GIRM to the one-dimensional problem with constant advection velocity and constant diffusion coefficient.

If $C(x, t)$ is substance density in the region $-L<x<L$, the corresponding IBVP is given by

Differential equation: 


$$
\frac{\partial C}{\partial t}+U \frac{\partial C}{\partial x}=\kappa \frac{\partial^{2} C}{\partial x^{2}}+\sigma,-L<x<L, t>0
$$

Boundary condition:

$$
C(-L, t)=g_{-L}(t), C(+L, t)=g_{+L}(t), t>0
$$

Initial condition:

$$
\begin{array}{r}
0=\int_{-L}^{+L}\left(\frac{\partial C(x, t)}{\partial t}+U \frac{\partial C(x, t)}{\partial x}-\kappa \frac{\partial^{2} C(x, t)}{\partial x^{2}}-\sigma(x)\right) \tilde{G}(x, \xi) d x=\int_{-L}^{+L} \frac{\partial C(x, t)}{\partial t} \tilde{G}(x, \xi) d x-U \int_{-L}^{+L} C(x, t) \frac{\partial \tilde{G}(x, \xi)}{\partial x} d x \\
-\kappa \int_{-L}^{+L} C(x, t) \frac{\partial^{2} \tilde{G}(x, \xi)}{\partial x^{2}} d x+U[C(x, t) \tilde{G}(x, \xi)]_{x=-L}^{x=+L}-\kappa\left[\frac{\partial C(x, t)}{\partial x} \tilde{G}(x, \xi)-C(x, t) \frac{\partial \tilde{G}(x, \xi)}{\partial x}\right]_{x=-L}^{x=+L}-\int_{-L}^{+L} \sigma(x, t) \tilde{G}(x, \xi) d x
\end{array}
$$

If we rewrite Eq. (4) and exchange $x$ and $\xi$, we have

$$
\begin{array}{r}
\int_{-L}^{+L} \frac{\partial C(\xi, t)}{\partial t} \tilde{G}(\xi, x) d \xi=U \int_{-L}^{+L} C(\xi, t) \frac{\partial \tilde{G}(\xi, x)}{\partial \xi} d \xi+\kappa \int_{-L}^{+L} C(\xi, t) \frac{\partial^{2} \tilde{G}(\xi, x)}{\partial \xi^{2}} d \xi-U[C(\xi, t) \tilde{G}(\xi, x)]_{\xi=-L}^{\xi+L}+\int_{-L}^{L} \sigma(\xi, t) \tilde{G}(\xi, x) d \xi \\
+\kappa\left[\frac{\partial C(\xi, t)}{\partial \xi} \tilde{G}(\xi, x)-C(\xi, t) \frac{\partial \tilde{G}(\xi, x)}{\partial \xi}\right]_{\xi=-L}^{\xi=+L}
\end{array}
$$

where $\tilde{G}(x, \xi)$ is a Generalized Fundamental Solution (GFS) chosen properly. For instance, we take Gaussian GFS:

$$
\tilde{G}(x, \xi)=\frac{1}{\sqrt{2 \pi} \gamma} \exp \left(-\frac{(x-\xi)^{2}}{2 \gamma^{2}}\right)
$$

Eq. (5) is a generalized integral representation of Eq. (1). This integral representation is applied to numerical solution. If $C(x, t)$ in $-L<x<L, C(-L, t)$ and $C(L, t)$ are known, then Eq. (5) is an integral equation with unknowns $\partial C(x, t) / \partial t$ in $-L<x<L, C_{x}(-L, t)$ and $C_{x}(L, t)$, where $\tilde{G}(x, \xi)$ is the kernel function. Namely, we are able to obtain $C(x, t)$ numerically, if we use for instance, the following procedure:

Let $C(x, t)$ be known at time $t \rightarrow$

Obtain $\partial C(x, t) / \partial t$ from Eq. (5) $\rightarrow$
Apply $C(x, t+d t)=C(x, t)+d t \partial C(x, t) / \partial t \rightarrow$

Increment time by $d t \rightarrow$ Repeat process.

\subsection{Numerical Experiment}

To begin with, we introduce for example, a regular mesh:

$$
\begin{gathered}
d x=d \xi=2 L / N, \\
x_{i}=\xi_{i}=-L+(i+0.5) d x, i=0,1, \ldots, N-1 \\
t_{n}=n d t, n=0,1, \ldots
\end{gathered}
$$

and denote

$$
C_{i}^{(n)}=C\left(x_{i}, t_{n}\right), \sigma_{i}^{(n)}=\sigma\left(x_{i}, t_{n}\right),\left[\frac{\partial C}{\partial t}\right]_{j}^{(n)}=\frac{\partial C\left(\xi_{j}, t_{n}\right)}{\partial t}
$$

We prepare the following approximations for discretization of Eq. (5)

$$
\begin{gathered}
\int_{-L}^{+L} \frac{\partial C\left(\xi, t_{n}\right)}{\partial t} \tilde{G}(x, \xi) d \xi=\sum_{j=0}^{N-1} \int_{\xi_{j}-d \xi / 2}^{\xi_{j}+d \xi / 2} \frac{\partial C\left(\xi, t_{n}\right)}{\partial t} \tilde{G}(x, \xi) d \xi=\sum_{j=0}^{N-1}\left[\frac{\partial C}{\partial t}\right]_{j}^{(n)} \int_{\xi_{j}-d \xi / 2}^{\xi_{j}+d \xi / 2} \tilde{G}(x, \xi) d \xi \\
\\
{\left[\frac{\partial C\left(\xi, t_{n}\right)}{\partial \xi} \tilde{G}(x, \xi)\right]_{\xi=-L}^{\xi=+L}=\frac{\partial C\left(+L, t_{n}\right)}{\partial \xi} \tilde{G}(x,+L)-\frac{\partial C\left(-L, t_{n}\right)}{\partial \xi} \tilde{G}(x,-L)} \\
\int_{-L}^{+L} \sigma\left(\xi, t_{n}\right) \tilde{G}(x, \xi) d \xi=\sum_{j=0}^{N-1} \int_{\xi_{j}-d \xi / 2}^{\xi_{j}+d \xi / 2} \sigma\left(\xi, t_{n}\right) \tilde{G}(x, \xi) d \xi=\sum_{j=0}^{N-1} \sigma_{j}^{(n)} \int_{\xi_{j}-d \xi / 2}^{\xi_{j}+d \xi / 2} \tilde{G}(x, \xi) d \xi \\
{\left[C\left(\xi, t_{n}\right) \frac{\partial \tilde{G}(x, \xi)}{\partial \xi}\right]_{\xi=-L}^{\xi=+L}=g_{+L}\left(t_{n}\right) \frac{\partial \tilde{G}(x,+L)}{\partial \xi}--g_{-L}\left(t_{n}\right) \frac{\partial \tilde{G}(x,-L)}{\partial \xi}}
\end{gathered}
$$


where $\partial C\left(\xi_{j}, t_{n}\right) / \partial t=C_{\xi}\left(\xi_{j}, t_{n}\right)$ etc.

Thus Eq. (5) can be discretized

$$
\begin{gathered}
\sum_{j=0}^{N-1}\left[\frac{\partial C}{\partial t}\right]_{j}^{(n)} \Gamma_{j}(x)-\kappa\left[\frac{\partial C\left(L, t_{n}\right)}{\partial \xi} \tilde{G}(L, x)-\frac{\partial C\left(-L, t_{n}\right)}{\partial \xi} \tilde{G}(-L, x)\right]=\kappa \sum_{j=0}^{N-1} C_{j}^{(n)} \Xi_{j}(x)+U \sum_{j=0}^{N-1} C_{j}^{(n)} \Lambda_{j}(x)+\sum_{j=0}^{N-1} \sigma_{j}^{(n)} \Gamma_{j}(x) \\
-U\left[g_{+L}\left(t_{n}\right) \tilde{G}(L, x)-g_{-L}\left(t_{n}\right) \tilde{G}(-L, x)\right]-\kappa\left[g_{+L}\left(t_{n}\right) \frac{\partial \tilde{G}(L, x)}{\partial \xi}-g_{-L}\left(t_{n}\right) \frac{\partial \tilde{G}(-L, x)}{\partial \xi}\right]
\end{gathered}
$$

where

$$
\begin{aligned}
& \Gamma_{j}(x)=\int_{\xi_{j}-d \xi / 2}^{\xi_{j}+d \xi / 2} \tilde{G}(x, \xi) d \xi \\
& \Lambda_{j}(x)=\int_{\xi_{j}-d \xi / 2}^{\xi_{j}+d \xi / 2} \frac{\partial \tilde{G}(\xi, x)}{\partial \xi} d \xi \\
& \Xi_{j}(x)=\int_{\xi_{j}-d \xi / 2}^{\xi_{j}+d \xi / 2} \frac{\partial^{2} \tilde{G}(\xi, x)}{\partial \xi^{2}} d \xi
\end{aligned}
$$

The unknowns in Eq. (12) are $[\partial C / \partial t]_{j}^{(n)}$, $j=0,1, \ldots, N-1, \partial C\left(-L, t_{n}\right) / \partial \xi$ and $\partial C\left(L, t_{n}\right) / \partial \xi$. Eq. (12) is satisfied at the interior points $x=x_{0}, x_{1}, \ldots, x_{N-1}$ as well as at the boundary points $x= \pm L$. Hence, we have $N+2$ equations for $N+2$ unknowns. Furthermore, if we use approximations

$$
\frac{\partial C\left(-L, t_{n}\right)}{\partial \xi}=\frac{2}{d \xi}\left(C\left(x_{0}, t_{n}\right)-C\left(-L, t_{n}\right)\right),
$$

$$
\frac{\partial C\left(+L, t_{n}\right)}{\partial \xi}=\frac{2}{d \xi}\left(C\left(+L, t_{n}\right)-C\left(x_{N-1}, t_{n}\right)\right)
$$

and satisfy Eq. (12) at the interior points, we have $N$ equations for $N$ unknowns.

Although GIRM is computationally complex and requires matrix inversion, its accuracy of the numerical results is high. It can be applied to an irregular mesh. If a computer code is properly composed and implemented, the computational load might be comparable with the Finite element Method (FEM).

Numerical examples of the GIRM are given below. The initial condition is exponential and given by

$$
C(x, 0)=\exp \left(-\frac{1}{2}\left(\frac{x}{L / 8}\right)^{2}\right)
$$

We assume that $L$ is large enough, and the boundary condition is specified as

$$
C( \pm L, t)=0
$$

The exact solution is given by

$$
C(x, t)=\frac{1}{2 \sqrt{\pi \kappa t}} \int_{-\infty}^{+\infty} \exp \left(-\frac{1}{2}\left(\frac{\xi}{L / 8}\right)^{2}\right) \exp \left(-\frac{(x-\xi-U t)^{2}}{4 \kappa t}\right) d \xi
$$

In order to reduce spurious oscillation in numerical solutions, it is effective to use a finer mesh, but it invites serious increase of computational resource. Therefore, if necessary, numerical damping

$$
-\frac{4 \alpha}{d x^{2}}\left[C_{i}^{(n)}-\frac{1}{4}\left(C_{i+1}^{(n)}+2 C_{i}^{(n)}+C_{i-1}^{(n)}\right)\right]
$$

is added to $C_{i}^{(n)}$ at every time step, where $\alpha$ is a damping constant. Moreover, if the discontinuity of the initial density causes serious numerical errors, it is effective to replace $C_{i}^{(0)}$ with a filtered value such as

$$
\frac{1}{4}\left(C_{i+1}^{(0)}+2 C_{i}^{(0)}+C_{i-1}^{(0)}\right)
$$

Finally, for the reduction of computation time, numerical integrals that include function $\mathrm{G}$ and its derivatives w.r.t $\xi$ in the right hand side of Eq. (5) are calculated in the neighborhood of $x_{i}$ only:

$$
\sum_{j=0}^{N-1} I(i, j) \approx \sum_{|i-j| \leq b d w} I(i, j)
$$

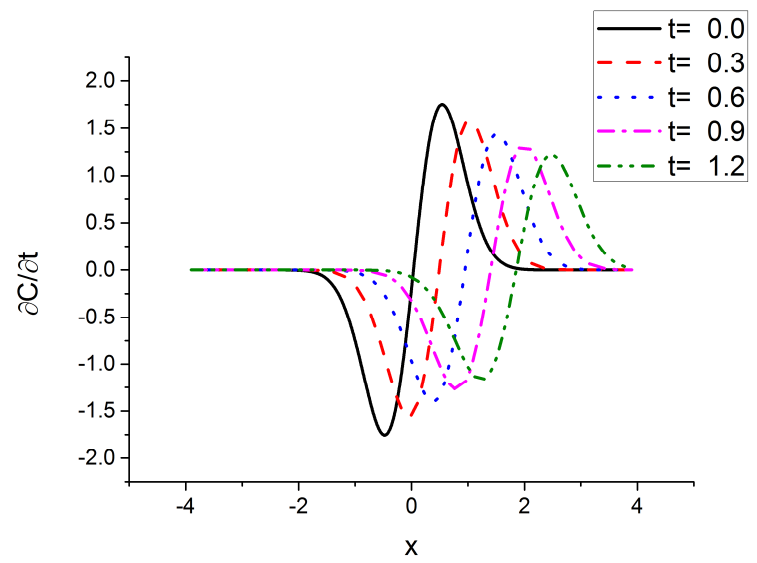

(a) $\partial \mathrm{C} / \partial \mathrm{t}$ 


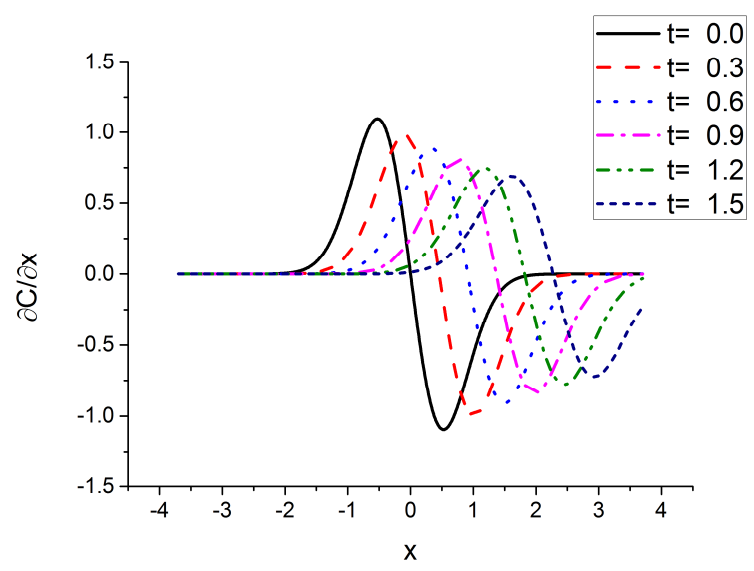

(b) $\partial \mathrm{C} / \partial \mathrm{x}$

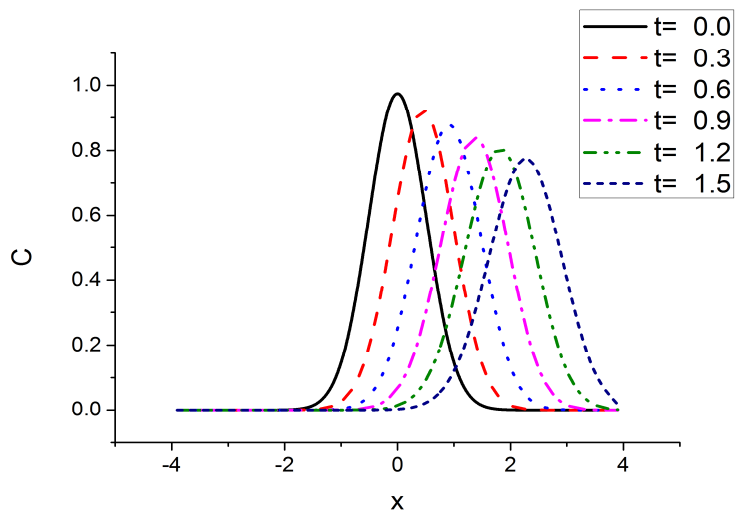

(c) $\mathrm{C}$
Numerical results are shown in Fig. 1. The accuracy of the numerical results is very high and it coincides with the exact ones. Values of the parameters used in the numerical experiment are:

$$
\begin{array}{r}
L=4, N=40, d x=2 L / 8=0.2, d t=0.0005, U=1.5, \\
T=3000 d t, \gamma=0.75 d x, \quad \kappa=0.05, N I t g=3 .
\end{array}
$$

The computer codes are given in Appendix A.

\section{Two-Step Generalized Integral Representation Method (2-Step GIRM)}

\subsection{Summary of Theory}

In order to derive two-step GIRM for Eq. (1), we rewrite it as follows:

$$
\begin{aligned}
& \text { Non-uniformity equation: } \theta=\frac{\partial C}{\partial x} \\
& \text { Constitutive equation: } q=-\kappa \theta
\end{aligned}
$$

Equilibrium equation: $\frac{\partial C}{\partial t}+U \frac{\partial C}{\partial x}=-\frac{\partial q}{\partial x}+\sigma$

Multiplying both sides of Eq. (22) by function $\tilde{G}(x, \xi)$ and integrating over $-L<x<L$, we obtain

Figure 1. Numerical solutions by 1-Step GIRM: (a) Time derivative $\partial C / \partial t$, (b) Space derivative $\partial C / \partial x$ and (c) Solution $C$ itself, where used exponential initial density distribution.

$$
0=\int_{-L}^{+L}\left[\theta(x, t)-\frac{\partial C(x, t)}{\partial x}\right] \tilde{G}(x, \xi) d x=\int_{-L}^{+L}[\tilde{G}(x, \xi) \theta(x, t)+C(x, t) \tilde{\delta}(x, \xi)] d x-[C(+L, t) \tilde{G}(+L, \xi)-C(-L, t) \tilde{G}(-L, \xi)]
$$

where

$$
\begin{gathered}
\frac{\partial \tilde{G}(x, \xi)}{\partial x}=\tilde{\delta}(x, \xi) \\
\int_{-L}^{+L} \tilde{G}(\xi, x) \theta(\xi, t) d \xi=-\int_{-L}^{+L} C(\xi, t) \tilde{\delta}(\xi, x) d \xi+[\tilde{G}(+L, x) C(+L, t)-\tilde{G}(-L, x) C(-L, t)]
\end{gathered}
$$
generalized integral representation for Eq. (22):

A generalized integral representation of Eq. (24) is obtained similarly:

$$
\begin{array}{r}
0=\int_{-L}^{+L} \tilde{G}(x, \xi)\left[\frac{\partial C(x, t)}{\partial t}+U \frac{\partial C(x, t)}{\partial x}+\frac{\partial q(x, t)}{\partial x}-\sigma(x, t)\right] d x=\int_{-L}^{+L} \tilde{G}(x, \xi) \frac{\partial C(x, t)}{\partial t} d x-U \int_{-L}^{+L} C(x, t) \delta(x, \xi) d x \\
+U[\tilde{G}(+L, \xi) C(+L, t)-\tilde{G}(-L, \xi) C(-L, t)]+[\tilde{G}(+L, \xi) q(+L, t)-\tilde{G}(-L, \xi) q(-L, t)]-\int_{-L}^{+L} q(x, t) \tilde{\delta}(x, \xi) d x-\int_{-L}^{+L} \sigma(x, t) \tilde{G}(x, \xi) d x(28)
\end{array}
$$

Rewriting Eq. (28) and exchanging $x$ and $\xi$, we obtain a generalized integral representation for Eq. (24):

$$
\begin{gathered}
\int_{-L}^{+L} \tilde{G}(\xi, x) \frac{\partial C(\xi, t)}{\partial t} d \xi=\int_{-L}^{+L}[U C(\xi, t)+q(\xi, t)] \tilde{\delta}(\xi, x) d \xi+\int_{-L}^{+L} \sigma(x, t) \tilde{G}(x, \xi) d x-[\tilde{G}(+L, x) q(+L, t)-\tilde{G}(-L, x) q(-L, t)] \\
-U[\tilde{G}(+L, x) C(+L, t)-\tilde{G}(-L, x) C(-L, t)]
\end{gathered}
$$


Hence, we are able to solve $C(x, t)$ numerically, if we use for example, the following procedure:

Let $C(x, t)$ be known $\rightarrow$ Obtain $\theta(x, t)$ from (27)

Obtain $q(x, t)$ from $(23) \rightarrow$ Obtain $\partial C(x, t) / \partial t$ from (29)

Then $C(x, t+d t)=C(x, t)+d t \partial C(x, t) / \partial t \rightarrow$

Add $d t$ to $t \rightarrow$ Repeat process.

\subsection{Numerical Experiment}

Numerical examples are given for the same problem as in case of the 1-Step GIRM. The initial and boundary conditions are given by Eq. (3) and Eq. (16), respectively. The exact solution is given by Eq. (17). Values of the parameters used in the numerical experiment are:

$$
\begin{gathered}
L=4, N=40, d x=2 L / 8=0.2, d t=0.0005, U=1.5, \\
T=3000 d t, \gamma=0.75 d x, \kappa=0.05, \quad N I t g=3 .
\end{gathered}
$$

Numerical results are shown Fig. 2. The accuracy of the numerical results is very high and it coincides with the exact ones. The corresponding computer codes are given in Appendix B.

\section{Characteristics of Generalized Fundamental Solutions (GFS)}

In the numerical experiments in 2.2 and 3.2, Gaussian GFS was used. However, Gaussian GFS causes some problems, when the boundary value is not zero $[3,4]$. The remedies are given in Ref. [3,4]. For example, $C^{0}$ GFS such as harmonic and exponential GFSs works well. If the non-zero boundary value problem is transformed into a zero boundary value problem, Gaussian GFS is very effective.

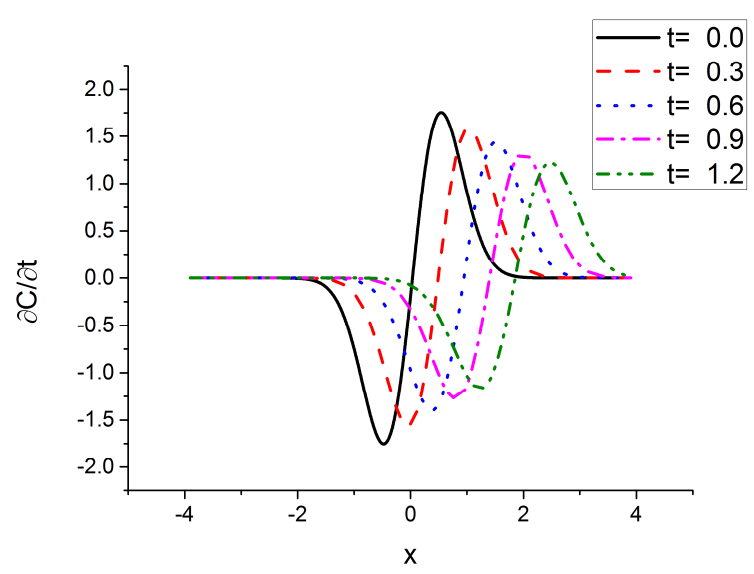

(a) $\partial \mathrm{C} / \partial \mathrm{t}$

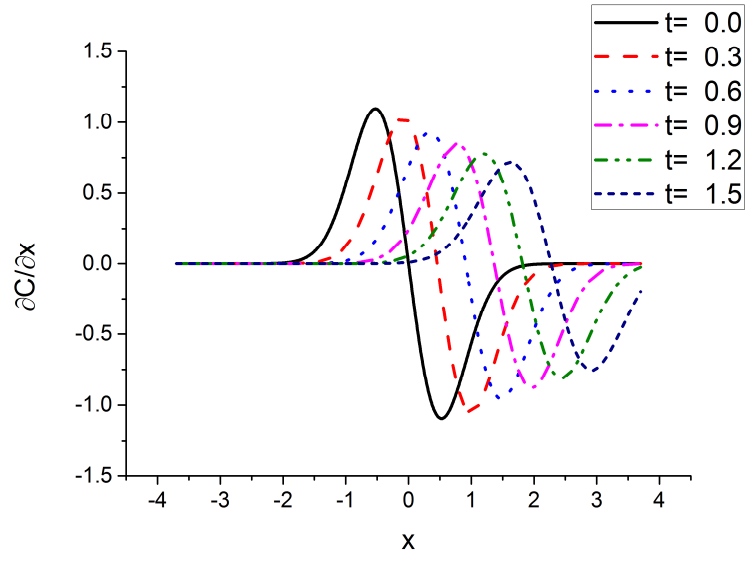

(b) $\partial \mathrm{C} / \partial \mathrm{x}$

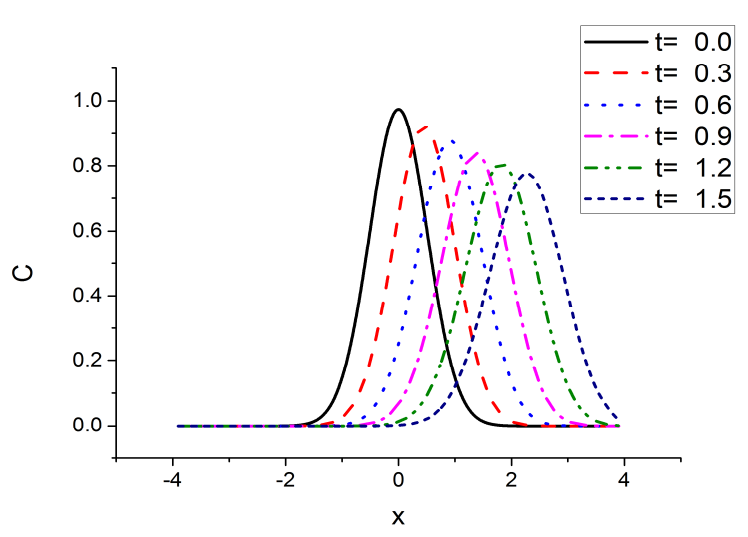

(c) $\mathrm{C}$

Figure 2. Numerical solutions by 2-Step GIRM: (a) Time derivative $\partial C / \partial t$, (b) Space derivative $\partial C / \partial x$ and (c) Solution $C$ itself, where used exponential initial density distribution.

\section{Conclusion}

GIRM is a convenient alternative to numerically solve the IBVP such as advective diffusion. Numerical solutions obtained by GIRM in particular, by 1- and 2-step GIRMs are stable and accurate.

In the present paper, we summarize and implement 1- and 2-step GIRMs. As implementation demonstration, we provide computer codes written in widely used computational languages including low-level programming languages such as $\mathrm{C}$ and FORTRAN as well as high-level language such as Matlab.

\section{Appendix A. Computer codes of 1-Step GIRM}

\section{A.1. Matlab code}

function GIRM_1_step ( )

$\%$ Solves 1D IBVP for advective diffusion using 1-step GIRM. 


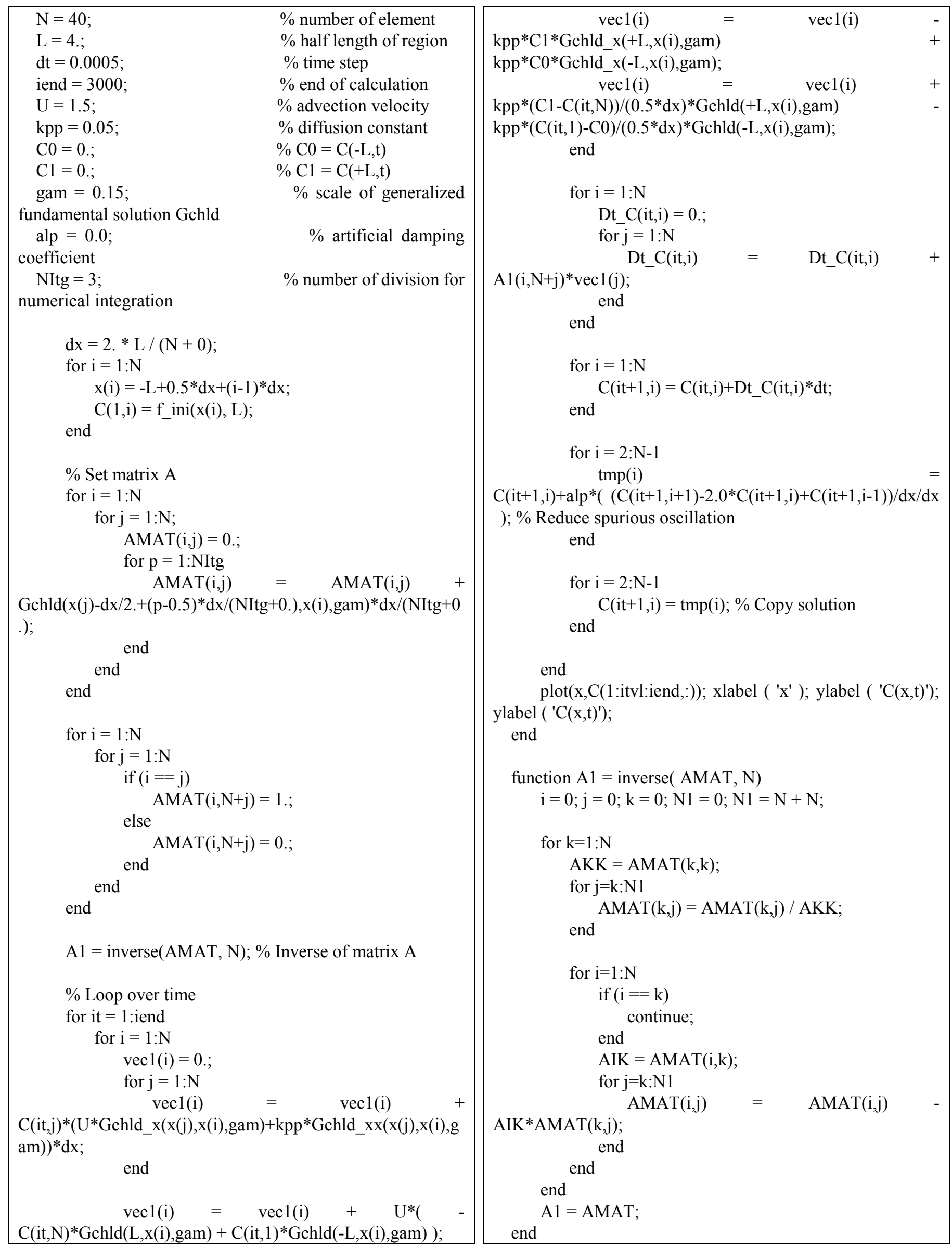




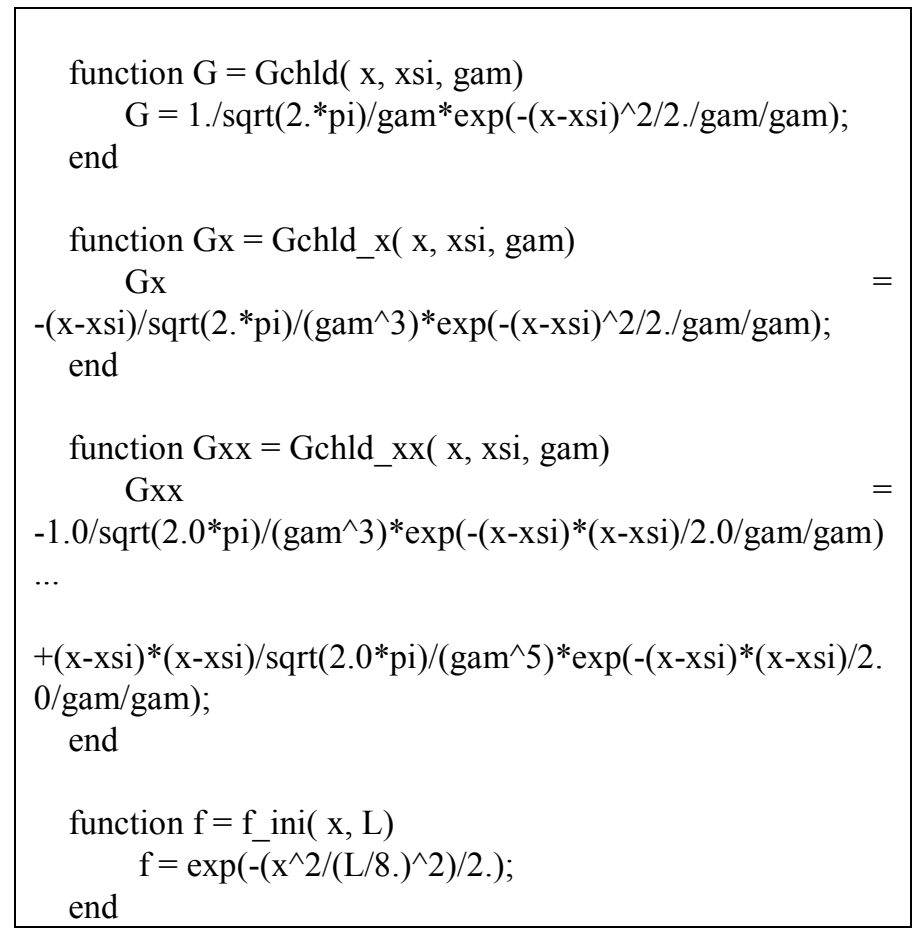

\section{A.2. C code}

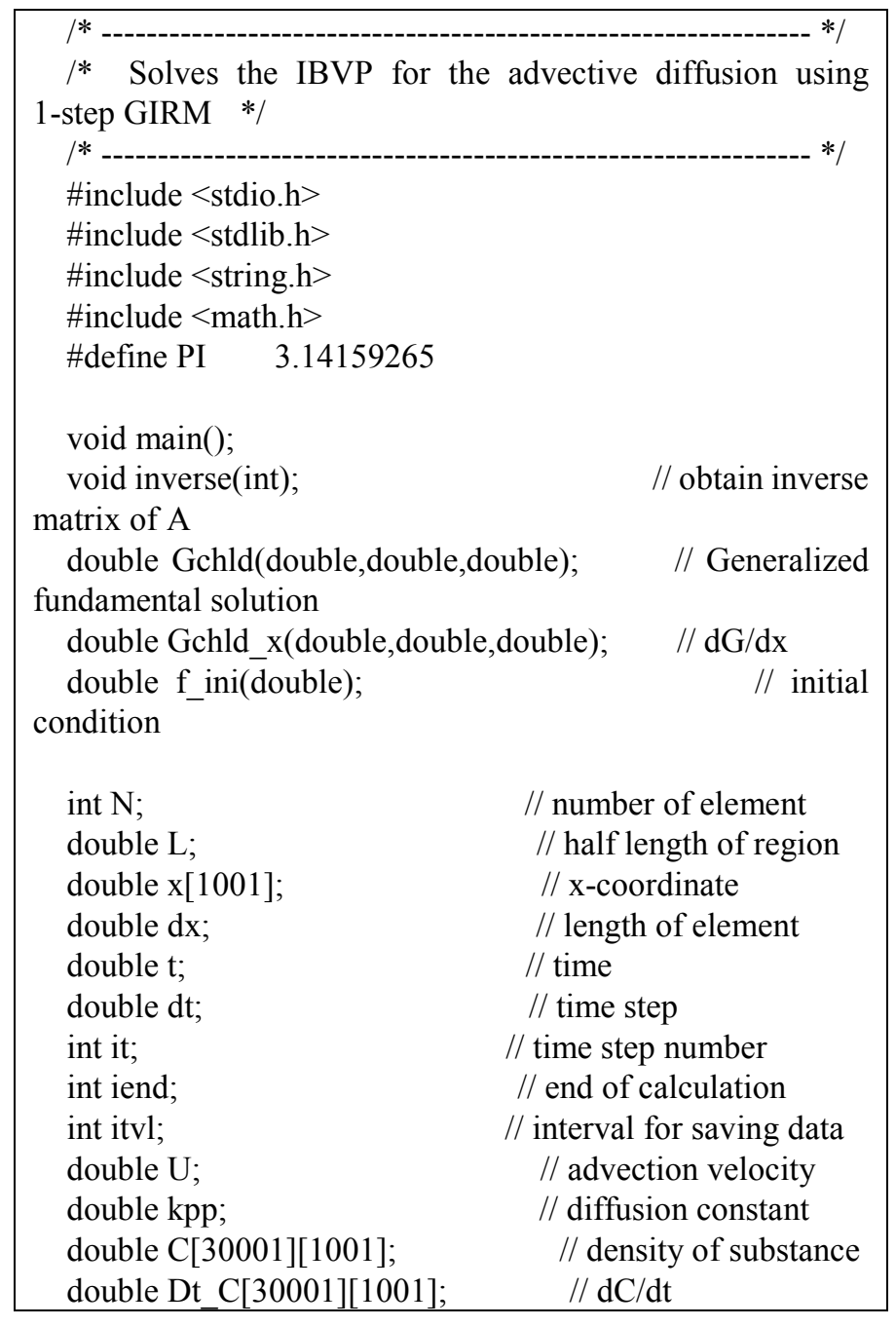

$/ / \mathrm{C} 0=\mathrm{C}(-\mathrm{L}, \mathrm{t})$

$/ / \mathrm{C} 1=\mathrm{C}(+\mathrm{L}, \mathrm{t})$

double $\mathrm{C} 1$;

// scale of generalized fundamental solution Gchld double alp;

// artificial damping coeff

int NItg;

numerical integration

double AMAT[1001][1001];

FILE *fp_inp;

FILE *fp_out;

char InputDataFile[80];

char OutputDataFile[80];

char buf[500];

double vec[1001];

double vec1[1001];

double tmp[401];

// number of division for

// matrix

$/ /$ pointer of input file

$/ /$ pointer of output file

// input file name

// output file name

// buffer for texts

// temporary vector

// temporary vector

// temporary array

$$
\text { void main() }
$$

int $\mathrm{i}, \mathrm{j}, \mathrm{p}$;

sprintf(InputDataFile, "GIRM1Step_inp.dat"); // Input file if $\left(\left(\mathrm{fp} \_\right.\right.$inp $=$fopen $($InputDataFile, $\left." r ")\right)==$ NULL $)\{$
printf("Failed in Reading Input Data File! ... \%s $\backslash n "$, InputDataFile);

$$
\text { exit(1); }
$$

\}

fscanf(fp_inp, "\%s \%lf", buf, \&kpp);

fscanf(fp inp, "\%s \%lf", buf, \&L);

fscanf(fp_inp, "\%s \%d", buf, \&N);

fscanf(fp_inp, "\%s \%lf", buf, \&C0);

fscanf(fp_inp, "\%s \%lf", buf, \&C1);

fscanf(fp_inp, "\%s \%lf", buf, \&dt);

fscanf(fp_inp, "\%s \%d", buf, \&iend);

fscanf(fp_inp, "\%s \%d", buf, \&itvl);

fscanf(fp_inp, "\%s \%lf", buf, \&U);

fscanf(fp_inp, "\%s \%lf", buf, \&gam);

fscanf(fp_inp, "\%s \%lf", buf, \&alp);

fscanf(fp_inp, "\%s \%d", buf, \&NItg);

fscanf(fp_inp, "\%s \%d", buf, \&ini_no);

fclose(fp_inp);

$\mathrm{dx}=2 * \mathrm{~L} /(\mathrm{N}+0.0)$

printf("kpp =\%12.6fln", kpp);

printf("L = \%12.6fln", L);

printf("N $=\% \mathrm{~d} \backslash \mathrm{n} ", \mathrm{~N})$;

printf("C0 =\%12.6fln", C0);

printf("C1 =\%12.6fln", C1);

printf("dx =\%12.6fln", dx);

printf("dt =\%12.6f(n", dt);

printf("iend $=\% \mathrm{~d} \backslash n "$, iend);

printf("itvl = \%d $\backslash n "$, itvl); 
printf("U =\%12.6fin", U);

printf("gam =\%12.6fln", gam);

printf("alp $=\% 12.6 \mathrm{~g} \backslash \mathrm{n} "$, alp);

printf("NItg $=\% \mathrm{~d} \backslash \mathrm{n} ", \mathrm{NItg})$;

printf("ini_no $=\% \mathrm{~d} \backslash n$ ", ini_no);

// Output file

sprintf(OutputDataFile, "GIRM1Step_out.csv");

if $\left(\left(\mathrm{fp} \_\right.\right.$out $=$fopen $($OutputDataFile, "w") $)==$NULL $)\{$ printf("Failed in Reading Output Data

File! ... \%s\n", OutputDataFile);

exit(1);

\}

fprintf(fp_out, "kpp =, \%12.6f $\backslash n ", k p p)$;

fprintf(fp_out, "L=, \%12.6f, $N=, \% d, d x=, \% 12.6 f \mathrm{ln} "$,

$\mathrm{L}, \mathrm{N}, \mathrm{dx}$ );

fprintf(fp_out, "C0 =, \%12.6f, C1 =, \%12.6fin", C0, C1);

fprintf(fp_out, $" \mathrm{dt}=, \% 12.6 \mathrm{f}$, iend $=, \% \mathrm{~d}, \mathrm{itvl}=, \% \mathrm{~d} \backslash \mathrm{n} "$, $\mathrm{dt}$, iend, itvl);

fprintf(fp_out, "U =, \%12.6fln", U);

fprintf(fp_out, "gam =, \%12.6fln", gam);

fprintf(fp_out, "alp =, \%12.6g\n", alp);

printf("NItg $=\% \mathrm{~d} \backslash n "$, NItg);

fprintf(fp_out, "ini_no $=, \% \mathrm{~d} \backslash \mathrm{n}$ ", ini_no);

fprintf(fp_out, "\n");

for $(\mathrm{i}=0 ; \mathrm{i}<\mathrm{N} ; \mathrm{i}++)\{$

$x[i]=-L+0.5 * d x+(i+0.0) * d x ;$

$\mathrm{C}[0][\mathrm{i}]=\mathrm{f} \operatorname{ini}(\mathrm{x}[\mathrm{i}])$;

\}

// inverse of matrix A

for $(\mathrm{i}=0 ; \mathrm{i}<\mathrm{N} ; \mathrm{i}++)$

for $(\mathrm{j}=0 ; \mathrm{j}<\mathrm{N} ; \mathrm{j}++)\{$

AMAT $[\mathrm{i}][\mathrm{j}]=0.0$;

for $(\mathrm{p}=0 ; \mathrm{p}<\mathrm{NItg} ; \mathrm{p}++)$

AMAT[i][j]

Gchld $(x[\mathrm{j}]-\mathrm{dx} / 2.0+(\mathrm{p}+0.5) * \mathrm{dx} /(\mathrm{NItg}+0.0), \mathrm{x}[\mathrm{i}]$,gam $) * \mathrm{dx} /(\mathrm{NItg}$ +0.0 )

$$
\begin{aligned}
& \text { for }(\mathrm{i}=0 ; \mathrm{i}<\mathrm{N} ; \mathrm{i}++) \\
& \text { for }(\mathrm{j}=0 ; j<\mathrm{j} ; \mathrm{j}++)\{ \\
& \quad \text { if }(\mathrm{i}==\mathrm{j}) \\
& \quad \operatorname{AMAT}[\mathrm{i}][\mathrm{N}+\mathrm{j}]=1.0 \\
& \text { else } \\
& \text { AMAT }[\mathrm{i}][\mathrm{N}+\mathrm{j}]=0.0
\end{aligned}
$$

inverse $(\mathrm{N}) ; \quad / /$ inverse of matrix A

for (it $=0$; it $<$ iend; it ++$)\{$

for $(\mathrm{i}=0 ; \mathrm{i}<\mathrm{N} ; \mathrm{i}++)\{$

$\operatorname{vec} 1[\mathrm{i}]=0.0$;

$$
\text { for }(j=0 ; j<N ; j++)
$$$$
\text { vec1[i] }
$$

\section{$+=$}

$\mathrm{C}[\mathrm{it}][\mathrm{j}] *\left(\mathrm{U}^{*} \mathrm{Gchld} \_\mathrm{x}(\mathrm{x}[\mathrm{j}], \mathrm{x}[\mathrm{i}], \mathrm{gam})+\mathrm{kpp} * \mathrm{Gchld} \mathrm{xx}(\mathrm{x}[\mathrm{j}], \mathrm{x}[\mathrm{i}]\right.$, gam) $) * d x$;

$\operatorname{vec} 1[\mathrm{i}]+=\mathrm{U} *(-\mathrm{C}[\mathrm{it}][\mathrm{N}-1] * \operatorname{Gchld}(\mathrm{L}, \mathrm{x}[\mathrm{i}]$,gam $)$

$+\mathrm{C}[\mathrm{it}][0] * \operatorname{Gchld}(-\mathrm{L}, \mathrm{x}[\mathrm{i}]$,gam $))$;

$\operatorname{vec} 1[\mathrm{i}]+=-\mathrm{kpp} * \mathrm{C} 1 * \mathrm{Gchld} \_\mathrm{x}(+\mathrm{L}, \mathrm{x}[\mathrm{i}], \mathrm{gam})+$ $\mathrm{kpp} * \mathrm{C} 0 *$ Gchld_x(-L, $\mathrm{x}[\mathrm{i}]$,gam $)$; $\operatorname{vec} 1[\mathrm{i}]$

$+\mathrm{kpp}^{*}(\mathrm{C} 1-\mathrm{C}[\mathrm{it}][\mathrm{N}-1]) /(0.5 * \mathrm{dx}) * \mathrm{Gchld}(+\mathrm{L}, \mathrm{x}[\mathrm{i}]$,gam $)$

kpp*(C[it] [0]-C0)/(0.5*dx)*Gchld(-L,x[i],gam);

\}

$$
\begin{aligned}
& \text { for }(i=0 ; i<N ; i++)\{ \\
& \text { Dt_C }[i t][i]=0.0 ; \\
& \text { for }(j=0 ; j<N ; j++) \\
& \quad \text { Dt_C }[i t][i]+=\operatorname{AMAT}[i][N+j]^{*} \operatorname{vec} 1[j]
\end{aligned}
$$$$
\text { \} }
$$

if (it $\% 20==0)\{$

printf("Solution it $=\% \mathrm{~d} \backslash \mathrm{n}$ ", it);

for $(\mathrm{i}=0 ; \mathrm{i}<\mathrm{N} ; \mathrm{i}++)$

$$
\text { printf("it } \quad=\quad \% 4 \mathrm{~d} \text {, }
$$

$=\% 4 d, \% 12.6 f, \% 12.6 f(n "$, it, i, Dt_C $[i t][i], C[i t][i])$;

\}

$$
\begin{aligned}
& \text { for }(i=0 ; i<N ; i++) \\
& \qquad C[i t+1][i]=C[i t][i]+D t \_C[i t][i] * d t ;
\end{aligned}
$$

// reduction of spurious oscillation

for $(\mathrm{i}=1 ; \mathrm{i}<\mathrm{N}-1 ; \mathrm{i}++)$

tmp[i]

$\mathrm{C}[\mathrm{it}+1][\mathrm{i}]+\mathrm{alp} *((\mathrm{C}[\mathrm{it}+1][\mathrm{i}+1]-2.0 * \mathrm{C}[\mathrm{it}+1][\mathrm{i}]+\mathrm{C}[\mathrm{it}+1][\mathrm{i}-1]) / \mathrm{d}$ $\mathrm{x} / \mathrm{dx})$;

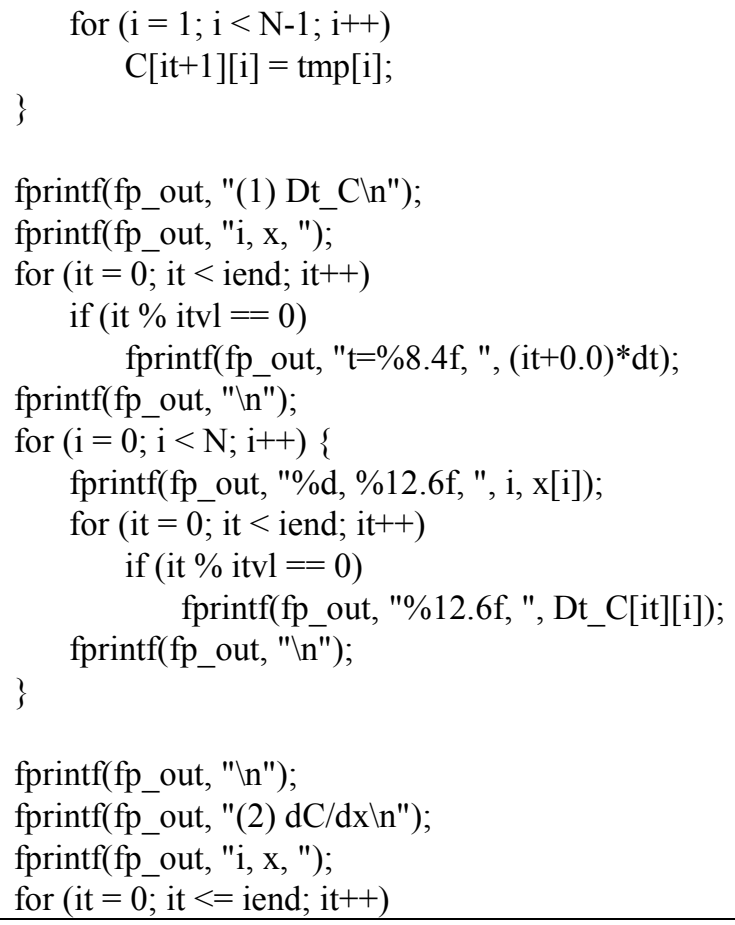




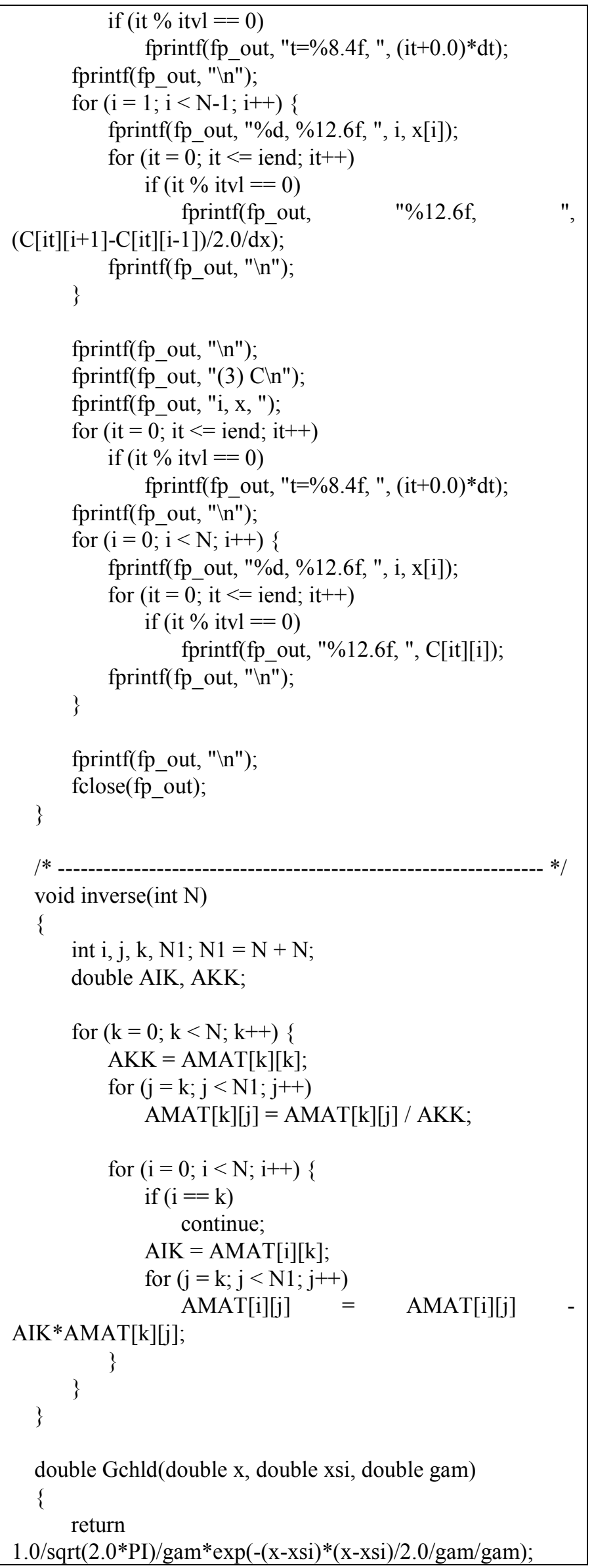

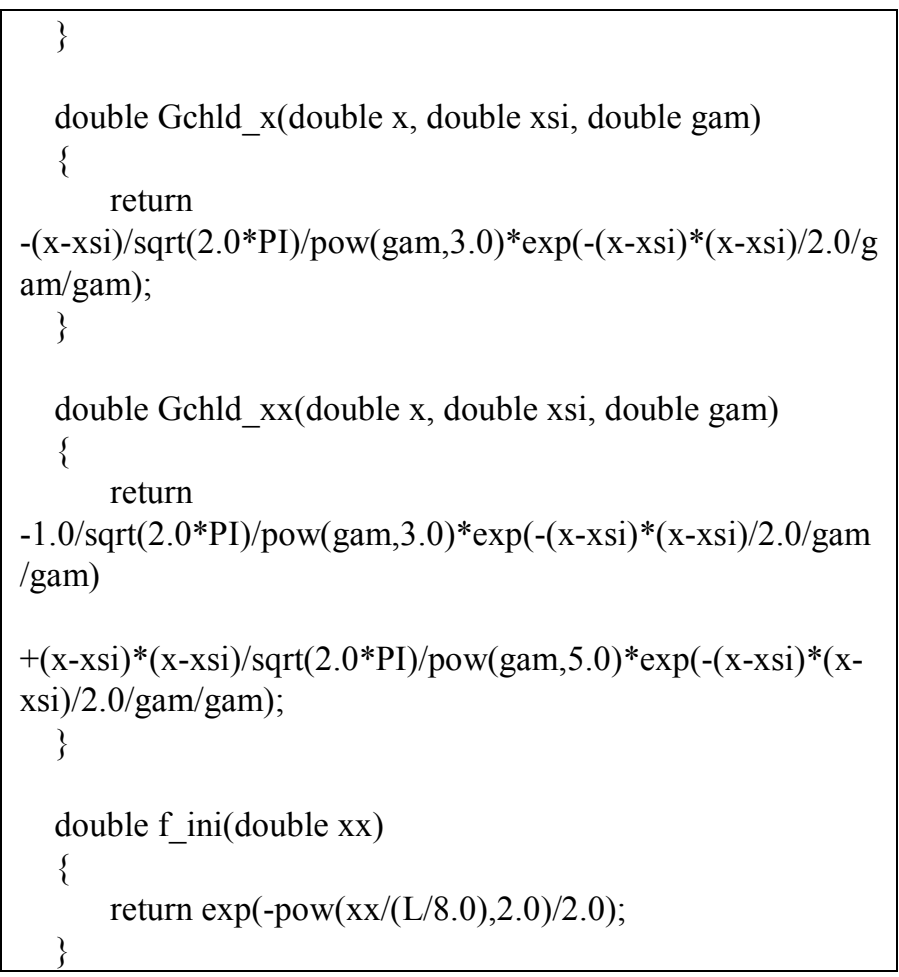

\section{A.3. FORTRAN code}

integer $\mathrm{n}, \mathrm{a}(100), \mathrm{m}, \mathrm{k}$

real*8 $\mathrm{L}, \mathrm{tt}(100)$

real*8 x(1001)

real*8 dx

real*8 t

real*8 dt

integer it, iend, itvl

real*8 u

real*8 kpp

real*8 c $(30001,1001)$

real*8 $\mathrm{dtc}(30001,1001)$

real*8 dcdx $(30001,1001)$

real*8 $\mathrm{c} 0, \mathrm{c} 1$

real*8 gam

real*8 alp

integer nitg

integer $\mathrm{i}, \mathrm{j}, \mathrm{p}$

real*8 amat $(1001,1001)$

real*8 vec $(1001)$

real*8 vec1(1001)

real*8 tmp (401)

real*8 sum

$\mathrm{c}$

open(11,file='input2.dat')

$\operatorname{open}\left(12\right.$, file $={ }^{\prime}$ calcu2.csv')

$\mathrm{c}$

$\operatorname{read}(11, *) \mathrm{kpp}$
$\operatorname{read}\left(11,{ }^{*}\right) \mathrm{L}$
$\operatorname{read}\left(11,{ }^{*}\right) \mathrm{n}$
$\operatorname{read}\left(11,{ }^{*}\right) \mathrm{c} 0$
$\operatorname{read}\left(11,{ }^{*}\right) \mathrm{c} 1$



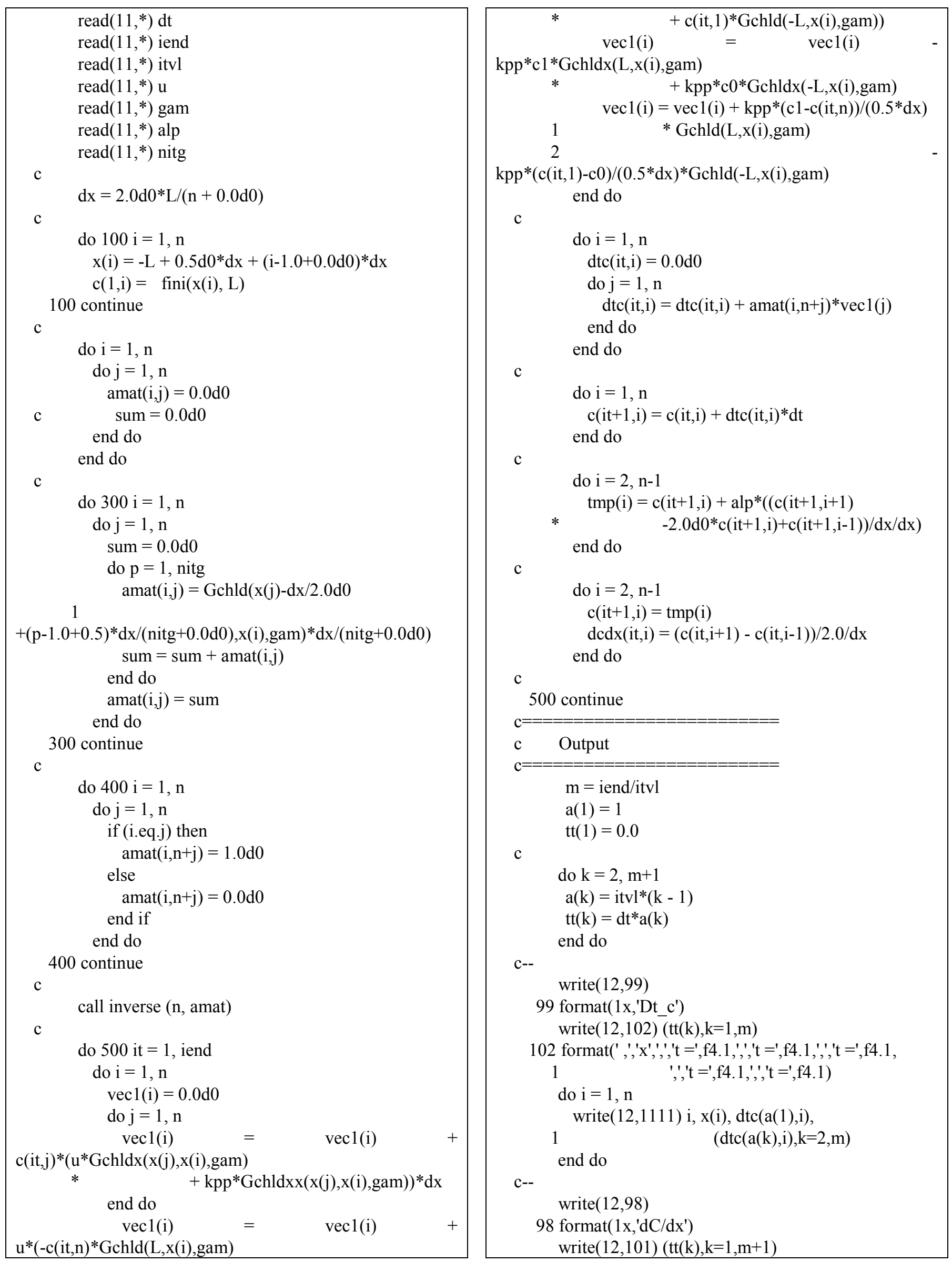


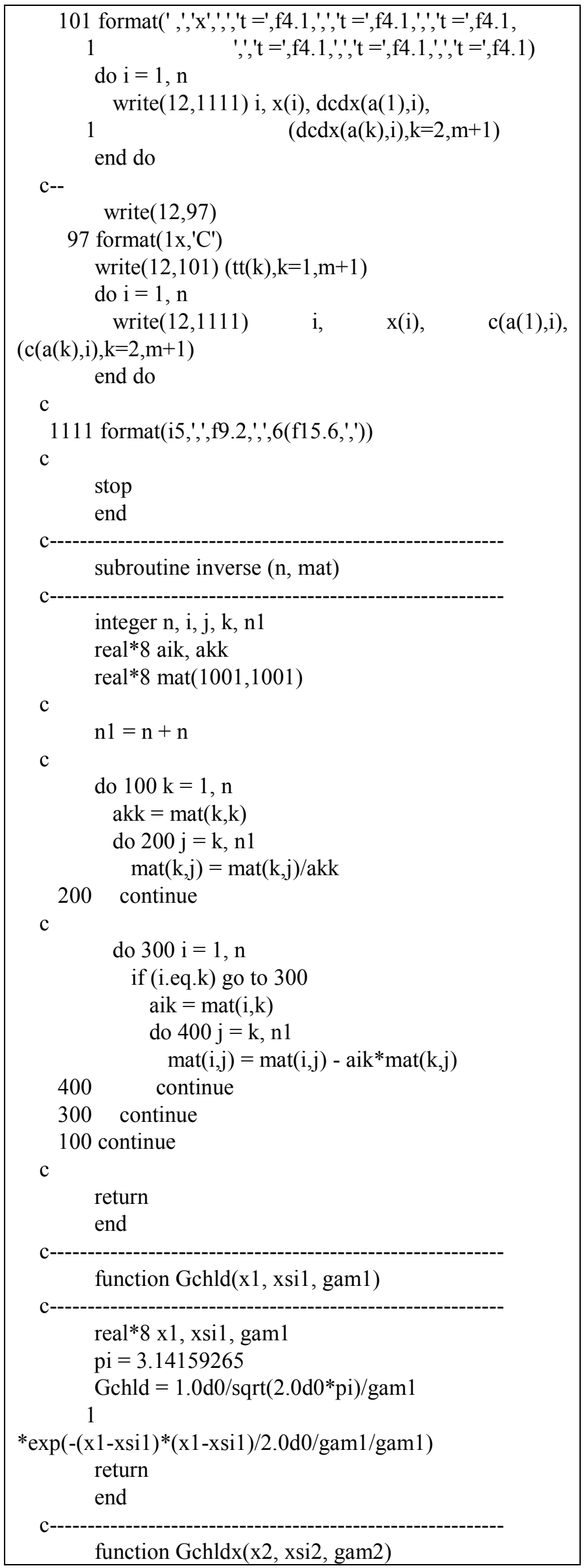

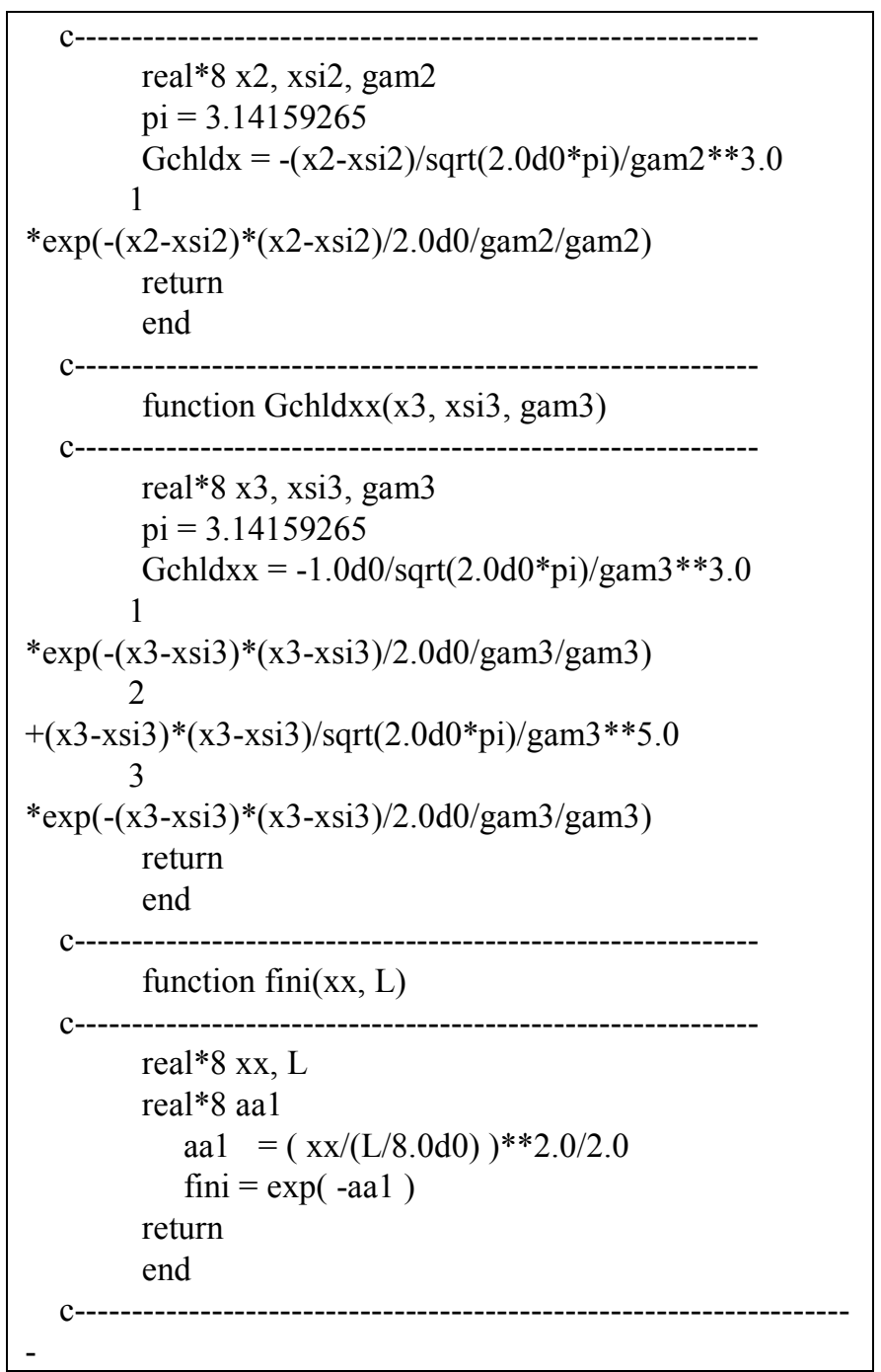

\section{Appendix B. Computer codes of 2-Step GIRM}

\section{B.1. Matlab code}

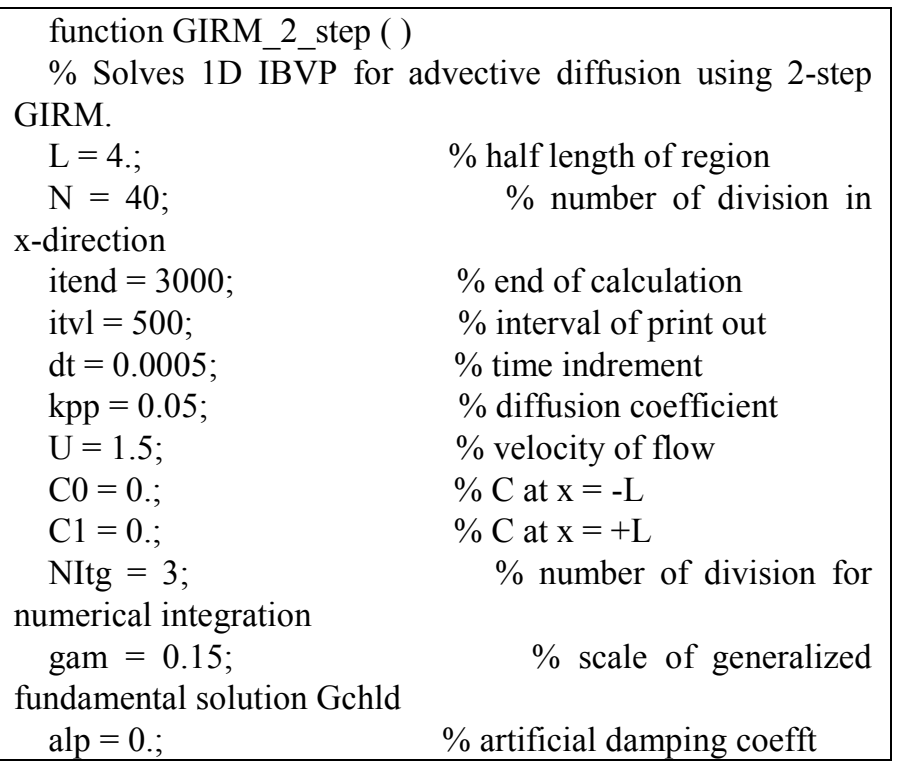




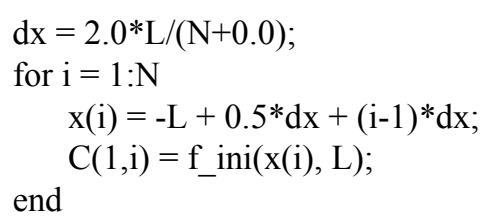

$\%$ Obtain inverse of kernel matrix A for $\mathrm{i}=1: \mathrm{N}$

$$
\begin{aligned}
& \text { for } \mathrm{n}=1: \mathrm{N} \\
& \operatorname{AMAT}(\mathrm{i}, \mathrm{n})=0.0 \text {; } \\
& \text { for } p=1: \text { NItg }
\end{aligned}
$$$$
\operatorname{AMAT}(\mathrm{i}, \mathrm{n}) \quad=\quad \operatorname{AMAT}(\mathrm{i}, \mathrm{n}) \quad+
$$

Gchld(x(n)-dx/2.0+(p-0.5)*dx/(NItg+0.0),x(i),gam)*dx/(NItg $+0.0)$;

$$
\begin{aligned}
& \text { end } \\
& \text { end }
\end{aligned}
$$$$
\text { for } \mathrm{i}=1: \mathrm{N}
$$$$
\text { for } \mathrm{j}=1: \mathrm{N}
$$$$
\text { if }(\mathrm{i}==\mathrm{j})
$$$$
\operatorname{AMAT}(\mathrm{i}, \mathrm{N}+\mathrm{j})=1 \text {; }
$$$$
\text { else }
$$$$
\operatorname{AMAT}(\mathrm{i}, \mathrm{N}+\mathrm{j})=0 ;
$$$$
\text { end }
$$$$
\text { end }
$$

end

$\%$ Inverse of matrix A

$\mathrm{A} 1$ = inverse $(\mathrm{AMAT}, \mathrm{N})$;

$\%$ Obtain solution at time $\mathrm{t}=\mathrm{it} * \mathrm{dt}$

for it $=1$ :itend

$$
\% \text { Obtain theta(i) at it }
$$

$$
\text { for } \mathrm{i}=1: \mathrm{N}
$$

$$
\begin{aligned}
& \operatorname{vec} 0(\mathrm{i})=0, \\
& \text { for } \mathrm{n}=1: \mathrm{N} \\
& \operatorname{vec} 0(\mathrm{i}) \quad=\quad \operatorname{vec} 0(\mathrm{i})
\end{aligned}
$$

$\mathrm{C}(\mathrm{it}, \mathrm{n}) * \mathrm{Gchld} \mathrm{x}(\mathrm{x}(\mathrm{n}), \mathrm{x}(\mathrm{i}), \mathrm{gam}) * \mathrm{dx}$;

$$
\text { end }
$$$$
\operatorname{vec} 0(\mathrm{i})=\operatorname{vec} 0(\mathrm{i})+\mathrm{C} 1 * \operatorname{Gchld}(+\mathrm{L}, \mathrm{x}(\mathrm{i}), \mathrm{gam})-
$$

C0*Gchld(-L,x(i),gam);

$$
\text { end }
$$

$$
\begin{aligned}
& \text { for } \mathrm{i}=1: \mathrm{N} \\
& \quad \operatorname{vec} 1(\mathrm{i})=0 . \\
& \text { for } \mathrm{j}=1: \mathrm{N} \\
& \quad \operatorname{vec} 1(\mathrm{i})=\operatorname{vec} 1(\mathrm{i})+\mathrm{A} 1(\mathrm{i}, \mathrm{N}+\mathrm{j}) * \operatorname{vec} 0(\mathrm{j}) \\
& \text { end } \\
& \text { end } \\
& \text { for } \mathrm{i}=1: \mathrm{N} \\
& \text { theta(i) }=\operatorname{vec} 1(\mathrm{i}) \text {; } \\
& \text { end }
\end{aligned}
$$

$\%$ Obtain qq(i) at it

$$
\text { for } \mathrm{i}=1: \mathrm{N}
$$

end

$$
\text { qq(i) }=-k p p * \text { theta(i); }
$$

$\%$ Obtain DtC at it

for $\mathrm{i}=1: \mathrm{N}$

$$
\begin{aligned}
& \operatorname{vec} 0(i)=0 ; \\
& \text { for } n=1: N
\end{aligned}
$$$$
\operatorname{vec} 0(\mathrm{i}) \quad=\quad \operatorname{vec} 0(\mathrm{i})
$$

$\mathrm{qq}(\mathrm{n}) * \operatorname{Gchld} \_\mathrm{x}(\mathrm{x}(\mathrm{n}), \mathrm{x}(\mathrm{i}), \mathrm{gam}) * \mathrm{dx}$;

end

$$
\text { for } \mathrm{n}=1: \mathrm{N}
$$

$$
\operatorname{vec} 0(\mathrm{i}) \quad=\quad \operatorname{vec} 0(\mathrm{i})
$$

$\mathrm{U} * \mathrm{C}(\mathrm{it}, \mathrm{n}) *$ Gchld_x$(\mathrm{x}(\mathrm{n}), \mathrm{x}(\mathrm{i}), \mathrm{gam}) * \mathrm{dx}$; end

$$
\operatorname{vec} 0(\mathrm{i}) \quad=\quad \operatorname{vec} 0(\mathrm{i})
$$

$2.0 * \mathrm{kpp}^{*}(\mathrm{C} 1-\mathrm{C}(\mathrm{it}, \mathrm{N})) / \mathrm{dx} * \mathrm{Gchld}(+\mathrm{L}, \mathrm{x}(\mathrm{i}), \mathrm{gam})$

$2.0 * \mathrm{kpp}^{*}(\mathrm{C}(\mathrm{it}, 1)-\mathrm{C} 0) / \mathrm{dx} * \mathrm{Gchld}(-\mathrm{L}, \mathrm{x}(\mathrm{i})$,gam $)$;

$$
\operatorname{vec} 0(\mathrm{i}) \quad=\quad \operatorname{vec} 0(\mathrm{i})
$$

$\mathrm{U}^{*} \mathrm{C}(\mathrm{it}, \mathrm{N}) * \mathrm{Gchld}(+\mathrm{L}, \mathrm{x}(\mathrm{i}), \mathrm{gam})$

$\mathrm{U}^{*} \mathrm{C}(\mathrm{it}, 1) *$ Gchld(-L, $\mathrm{x}(\mathrm{i})$,gam);

end

$$
\begin{aligned}
& \text { for } \mathrm{i}=1: \mathrm{N} \\
& \quad \operatorname{vec} 1(\mathrm{i})=0 \\
& \quad \text { for } \mathrm{j}=1: \mathrm{N} \\
& \quad \operatorname{vec} 1(\mathrm{i})=\operatorname{vec} 1(\mathrm{i})+\mathrm{A} 1(\mathrm{i}, \mathrm{N}+\mathrm{j}) * \operatorname{vec} 0(\mathrm{j}) \\
& \text { end }
\end{aligned}
$$

end

$$
\text { for } \mathrm{i}=1: \mathrm{N}
$$

end

$$
\operatorname{DtC}(i)=\operatorname{vec} 1(i)
$$

$\%$ Obtain $\mathrm{C}$ at it $=\mathrm{it}+1$

$$
\text { for } \mathrm{i}=1: \mathrm{N}
$$

$$
\mathrm{C}(\mathrm{it}+1, \mathrm{i})=\mathrm{C}(\mathrm{it}, \mathrm{i})+\mathrm{DtC}(\mathrm{i}) * \mathrm{dt} \text {; }
$$

end

$\%$ Reduction of spurious oscillation for $\mathrm{i}=2: \mathrm{N}-1$

$$
\operatorname{tmp}(\mathrm{i})
$$

$\mathrm{C}(\mathrm{it}+1, \mathrm{i})+\mathrm{alp} *((\mathrm{C}(\mathrm{it}+1, \mathrm{i}+1)-2.0 * \mathrm{C}(\mathrm{it}+1, \mathrm{i})+\mathrm{C}(\mathrm{it}+1, \mathrm{i}-1)) / \mathrm{dx} / \mathrm{dx}$ );

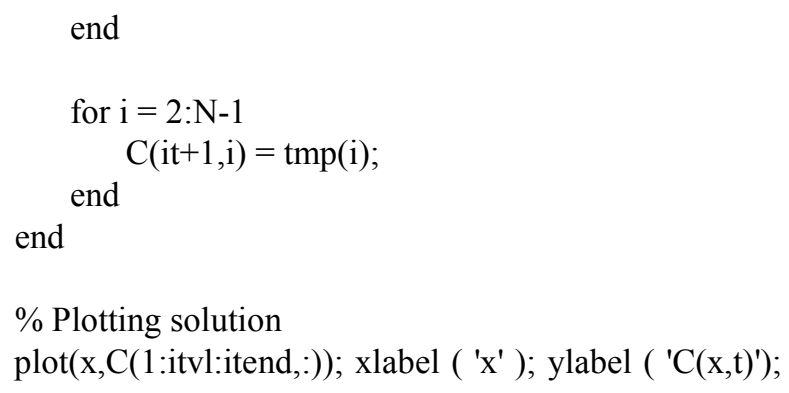




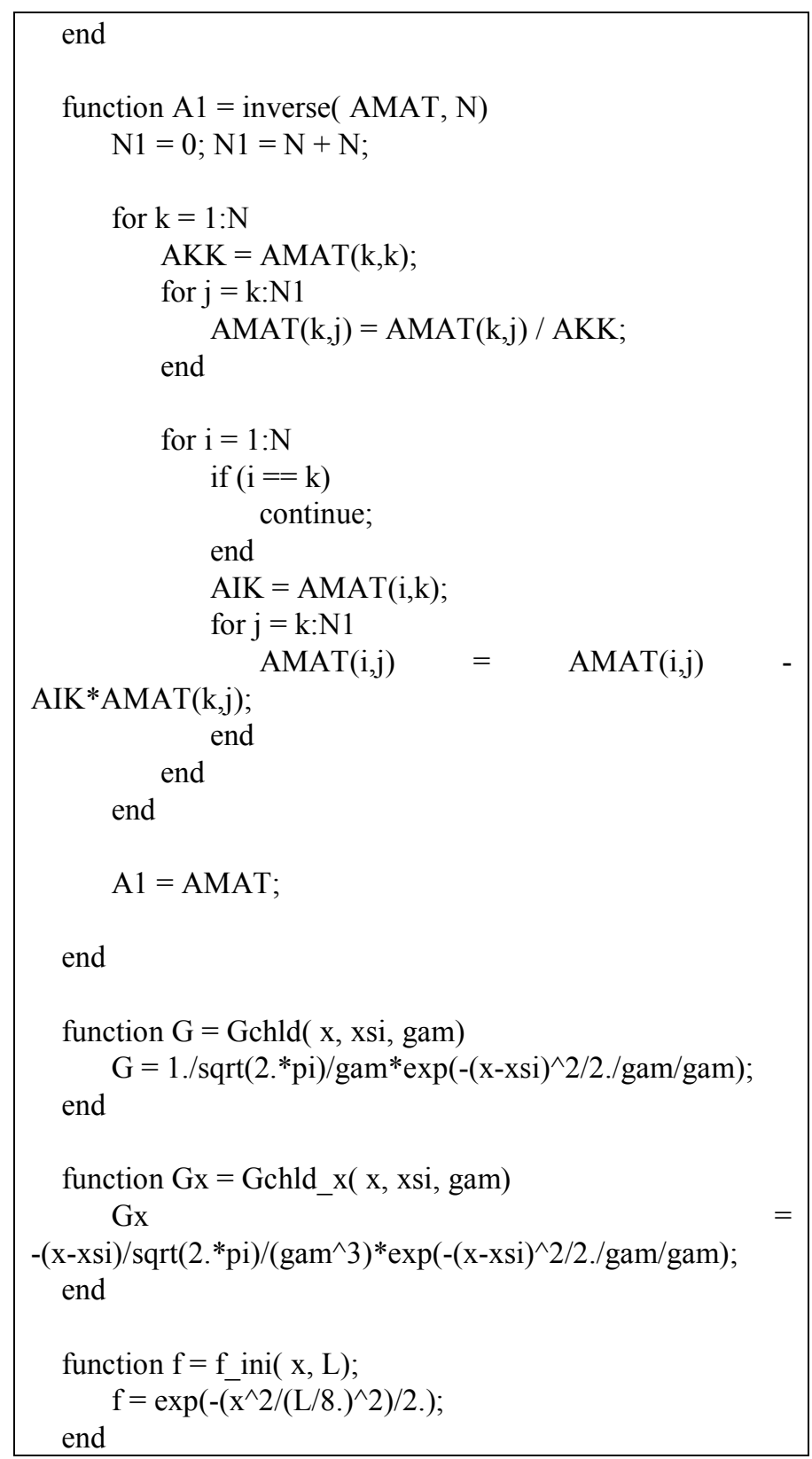

\section{B.2. C code}

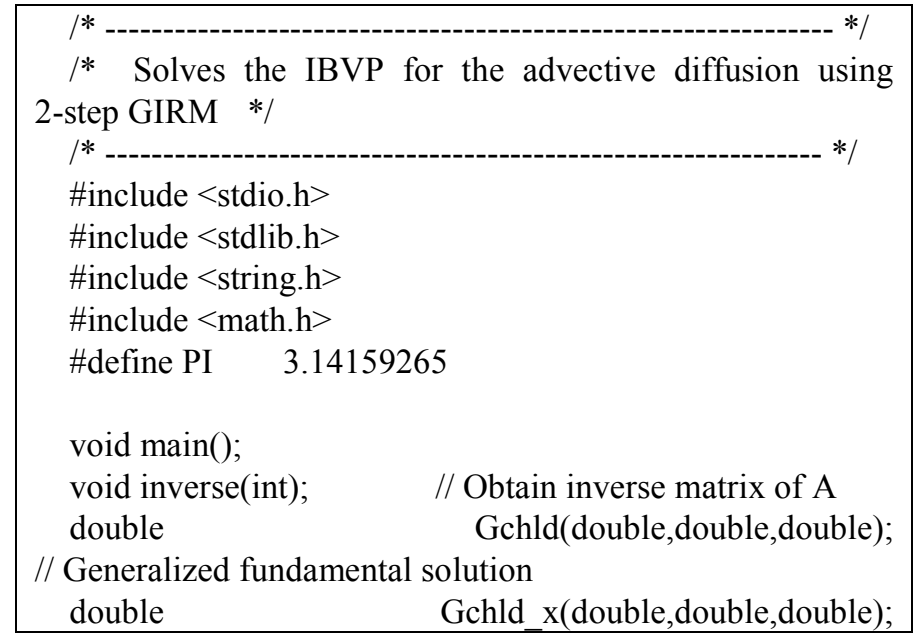

$/ / \mathrm{dG} / \mathrm{dx}$

double

// initial condition

double L;

int $\mathrm{N}$;

$\mathrm{x}$-direction

double $\mathrm{x}[401]$;

element center $-\mathrm{L}<\mathrm{x}<\mathrm{L}$

double $\mathrm{dx}$;

int it;

int itend;

int itvl;

double t;

double dt;

int itvl0;

double kpp;

double U;

double $\mathrm{C} 0$;

double $\mathrm{C} 1$;

int NItg;

numerical integration

double C[401];

double DtC[401];

double C_itvl0[1000][401];

double DtC_itv10[1000][401];

double theta[401];

double qq[401];

double theta_itv10[1000][401];

double gam;

fundamental solution Gchld

double alp;

coefft

double AMAT[4001][8001];

// half length of region

// number of division in

// x-coordinate of

// L/N

$/ /$ time step

// end of calculation

// interval of print out

// time

// time indrement

// interval of saving $\mathrm{C}$ etc.

// diffusion coefficient

$/ /$ velocity of flow

$/ / \mathrm{C}$ at $\mathrm{x}=-\mathrm{L}$

$/ / \mathrm{C}$ at $\mathrm{x}=+\mathrm{L}$

// number of divion for

// density of substance

$/ / \mathrm{dw} / \mathrm{dt}$

// $\mathrm{C}$ to be saved

// DtC to be saved

$/ / \mathrm{dC} / \mathrm{dx}$

$/ / \mathrm{qq}[\mathrm{i}]=-\mathrm{kpp} *$ theta[i]

// theta to be saved

// scale of generalized

// artificial damping

// matrix

double vec0[2001];

double vec1[2001];

FILE *fp_inp;

FILE *fp_out;

char InputDataFile[80];

char OutputDataFile[80];

char buf[500];

double tmp[401];

// vector

$/ /$ vector

$/ /$ pointer of input file

// pointer of output file

// input file name

// output file name

// buffer for texts

// temporary array

void main()

\{

int i, j, n, p;

// Input file

sprintf(InputDataFile, "GIRM2Step_inp.dat");

if $\left(\left(\mathrm{fp} \_\right.\right.$inp $=$fopen(InputDataFile, $\left.\left." \mathrm{r} "\right)\right)==$ NULL $)\{$ printf("Failed in Reading Input Data File! ... \%s $\backslash n$ ", InputDataFile); exit(1); 
fscanf(fp_inp, "\%s \%lf", buf, \&L); fscanf(fp_inp, "\%s \%d", buf, \&N); fscanf(fp_inp, "\%s \%lf", buf, \&kpp); fscanf(fp_inp, "\%s \%lf", buf, \&U); fscanf(fp_inp, "\%s \%lf", buf, \&C0); fscanf(fp_inp, "\%s \%lf", buf, \&C1); fscanf(fp_inp, "\%s \%lf", buf, \&dt); fscanf(fp_inp, "\%s \%d", buf, \&itend); fscanf(fp_inp, "\%s \%d", buf, \&itvl); fscanf(fp_inp, "\%s \%d", buf, \&itvl0); fscanf(fp_inp, "\%s \%lf", buf, \&gam); fscanf(fp_inp, "\%s \%lf", buf, \&alp); fscanf(fp_inp, "\%s \%d", buf, \&NItg); fscanf(fp_inp, "\%s \%d", buf, \&ini_no);

fclose(fp_inp);

$\mathrm{dx}=2.0 * \mathrm{~L} /(\mathrm{N}+0.0)$;

for $(\mathrm{i}=0 ; \mathrm{i}<\mathrm{N} ; \mathrm{i}++)\{$

$x[i]=-L+0.5 * d x+(i+0.0) * d x ;$

\}

$\mathrm{C}[\mathrm{i}]=\mathrm{f}$ _ini( $\mathrm{x}[\mathrm{i}]$, ini_no);

$\operatorname{printf("L\quad =\% 12.6f(n",~L);~}$

printf("N $\quad=\% \mathrm{~d} \backslash \mathrm{n} ", \mathrm{~N})$;

printf("kpp $=\% 12.6 \mathrm{fln} ", \mathrm{kpp})$;

printf("U = $=\% 12.6 \mathrm{f} \backslash \mathrm{n} ", \mathrm{U})$;

printf("C0 =\%12.6f $1 \mathrm{n}$ ", C0);

printf("C1 =\%12.6fln", C1);

printf("dx $=\% 12.6 f(n ", d x)$;

printf("dt $=\% 12.6 \mathrm{fln} ", \mathrm{dt})$;

printf("itend $=\% \mathrm{~d} \backslash \mathrm{n}$ ", itend);

printf("itvl =\%d 1 n", itvl);

printf("itvl0 =\%d $\backslash$ n", itvl0);

printf("gam =\%12.6fาn", gam);

printf("alp $=\% 12.6 \mathrm{~g} \backslash \mathrm{n} "$, alp);

printf("NItg $=\% \mathrm{~d} \backslash n "$, NItg);

printf("ini_no $=\% \mathrm{~d} \backslash n$ ", ini_no);

\section{// Output file}

sprintf(OutputDataFile,

"BasicProb_Gauss2Step_out.csv");

if $\left(\left(\mathrm{fp} \_\right.\right.$out $=$fopen $($OutputDataFile, "w") $)==$NULL $)\{$ printf("Failed in Reading Output Data

File! ... \%s\n", OutputDataFile); exit(1); \}

// input data

fprintf(fp_out, "L=, \%12.6fln", L);

fprintf(fp_out, "N =, \%d, dx =, \%12.6fln", N, dx);

fprintf(fp_out, "kpp =, \%12.6fln", kpp);

fprintf(fp_out, "U =, \%12.6fin", U);

C1);

fprintf(fp_out, "C0 =, \%12.6f, C1 =, \%12.6fln", C0,

fprintf(fp_out, "dt =, \%12.6fln", dt); fprintf(fp_out, "itend $=, \% d$, itvl $=, \% d$, itvl0 $=, \% d \backslash n "$, itend, itvl, itvl0);

fprintf(fp_out, "gam =, \%12.6fln", gam);

fprintf(fp_out, "alp =, \%12.6g\n", alp);

fprintf(fp_out, "NItg =, \%d $\backslash n$ ", NItg);

fprintf(fp_out, "ini_no $=, \% \mathrm{~d} \backslash \mathrm{n}$ ", ini_no);

fprintf(fp_out, "\n");

// obtain inverse of kernel matrix A

for $(\mathrm{i}=0 ; \mathrm{i}<\mathrm{N} ; \mathrm{i}++)$

$$
\begin{aligned}
& \text { for }(\mathrm{n}=0 ; \mathrm{n}<\mathrm{N} ; \mathrm{n}++)\{ \\
& \quad \operatorname{AMAT}[\mathrm{i}][\mathrm{n}]=0.0 ; \\
& \quad \text { for }(\mathrm{p}=0 ; \mathrm{p}<\mathrm{NItg} ; \mathrm{p}++) \\
& \quad \operatorname{AMAT}[\mathrm{i}][\mathrm{n}]
\end{aligned}
$$

$\operatorname{Gchld}(\mathrm{x}[\mathrm{n}]-\mathrm{dx} / 2.0+(\mathrm{p}+0.5) * \mathrm{dx} /(\mathrm{NItg}+0.0), \mathrm{x}[\mathrm{i}], \mathrm{gam}) * \mathrm{dx} /(\mathrm{NIt}$ $\mathrm{g}+0.0)$;

$$
\begin{aligned}
& \text { for }(i=0 ; i<N ; i++) \\
& \text { for }(j=0 ; j<N ; j++)\{ \\
& \quad \text { if }(i==j) \\
& \quad \operatorname{AMAT}[i][N+j]=1.0 ; \\
& \text { else } \operatorname{AMAT}[i][N+j]=0.0 ; \\
& \text { T }
\end{aligned}
$$

inverse(N);

for $(\mathrm{i}=1 ; \mathrm{i}<\mathrm{N}-1 ; \mathrm{i}++)$

theta_itvl0[0][i] $=(\mathrm{C}[\mathrm{i}+1]-\mathrm{C}[\mathrm{i}-1]) / 2.0 / \mathrm{dx}$;

for $(\mathrm{i}=0 ; \mathrm{i}<\mathrm{N} ; \mathrm{i}++)$

$$
\text { C_itvl0[0][i] = C }[\mathrm{i}] \text {; }
$$

// obtain solution at time $\mathrm{t}=\mathrm{it} * \mathrm{dt}$

for (it $=0$; it $<$ itend; it ++ ) \{

$$
\begin{aligned}
& / / \text { obtain theta[i] at it } \\
& \text { for }(\mathrm{i}=0 ; \mathrm{i}<\mathrm{N} ; \mathrm{i}++)\{ \\
& \operatorname{vec} 0[\mathrm{i}]=0.0 ; \\
& \text { for }(\mathrm{n}=0 ; \mathrm{n}<\mathrm{N} ; \mathrm{n}++) \\
& \quad \operatorname{vec} 0[\mathrm{i}]
\end{aligned}
$$

$-\mathrm{C}[\mathrm{n}] *$ Gchld_x(x[n],x[i],gam $) * d x$;

$\operatorname{vec} 0[\mathrm{i}]$

$+\mathrm{C} 1 * \operatorname{Gchld}(+\mathrm{L}, \mathrm{x}[\mathrm{i}]$,gam $)-\mathrm{C} 0 * \operatorname{Gchld}(-\mathrm{L}, \mathrm{x}[\mathrm{i}]$,gam $)$; \}

$$
\begin{aligned}
& \text { for }(i=0 ; i<N ; i++)\{ \\
& \quad \operatorname{vec} 1[i]=0.0 ; \\
& \text { for }(j=0 ; j<N ; j++) \\
& \quad \operatorname{vec} 1[i]+=\operatorname{AMAT}[i][N+j]^{*} \operatorname{vec} 0[j] \\
& \} \\
& \text { for }(i=0 ; i<N ; i++) \\
& \quad \text { theta }[i]=\operatorname{vec} 1[i]
\end{aligned}
$$


// obtain qq[i] at it

for $(\mathrm{i}=0 ; \mathrm{i}<\mathrm{N} ; \mathrm{i}++)$

$\mathrm{qq}[\mathrm{i}]=-\mathrm{kpp} * \operatorname{theta}[\mathrm{i}]$;

// obtain DtC at it

for $(\mathrm{i}=0 ; \mathrm{i}<\mathrm{N} ; \mathrm{i}++)\{$

$\operatorname{vec} 0[\mathrm{i}]=0.0$;

for $(\mathrm{n}=0 ; \mathrm{n}<\mathrm{N} ; \mathrm{n}++)$ $\operatorname{vec} 0[\mathrm{i}]$

$\mathrm{qq}[\mathrm{n}] *$ Gchld_x$(\mathrm{x}[\mathrm{n}], \mathrm{x}[\mathrm{i}], \mathrm{gam}) * \mathrm{dx}$;

$$
\begin{aligned}
& \text { for }(\mathrm{n}=0 ; \mathrm{n}<\mathrm{N} ; \mathrm{n}++) \\
& \quad \operatorname{vec} 0[\mathrm{i}]
\end{aligned}
$$

$\mathrm{U}^{*} \mathrm{C}[\mathrm{n}] *$ Gchld_x$(\mathrm{x}[\mathrm{n}], \mathrm{x}[\mathrm{i}], \mathrm{gam}) * \mathrm{dx}$;

$\operatorname{vec} 0[\mathrm{i}]$

$2.0 * \mathrm{kpp}^{*}(\mathrm{C} 1-\mathrm{C}[\mathrm{N}-1]) / \mathrm{dx} * \mathrm{Gchld}(+\mathrm{L}, \mathrm{x}[\mathrm{i}]$,gam $)$

$2.0 * \mathrm{kpp}^{*}(\mathrm{C}[0]-\mathrm{C} 0) / \mathrm{dx} * \operatorname{Gchld}(-\mathrm{L}, \mathrm{x}[\mathrm{i}]$,gam $)$;

$\operatorname{vec} 0[\mathrm{i}]+=-\mathrm{U}^{*} \mathrm{C}[\mathrm{N}-1] * \operatorname{Gchld}(+\mathrm{L}, \mathrm{x}[\mathrm{i}]$,gam $)+$ $\mathrm{U} * \mathrm{C}[0]^{*} \operatorname{Gchld}(-\mathrm{L}, \mathrm{x}[\mathrm{i}]$,gam $)$;

\}

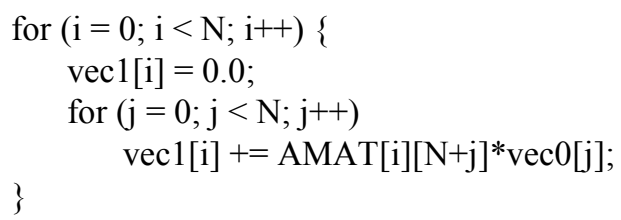

// Reduction of spurious oscillation

for $(\mathrm{i}=1 ; \mathrm{i}<\mathrm{N}-1 ; \mathrm{i}++)$

tmp[i]

$\mathrm{C}[\mathrm{i}]+\mathrm{alp}^{*}((\mathrm{C}[\mathrm{i}+1]-2.0 * \mathrm{C}[\mathrm{i}]+\mathrm{C}[\mathrm{i}-1]) / \mathrm{dx} / \mathrm{dx})$;

for $(\mathrm{i}=1 ; \mathrm{i}<\mathrm{N}-1 ; \mathrm{i}++)$

$\mathrm{C}[\mathrm{i}]=\operatorname{tmp}[\mathrm{i}]$;

$$
\begin{aligned}
& \text { if }((\mathrm{it}+0) \% \operatorname{itvl0}==0) \\
& \text { for }(\mathrm{i}=1 ; \mathrm{i}<\mathrm{N}-1 ; \mathrm{i}++) \\
& \text { DtC_itvl0[(it+0)/itvl0][i] = DtC }[\mathrm{i}] ;
\end{aligned}
$$

if $((\mathrm{it}+1) \%$ itvl0 $==0)\{$

for $(\mathrm{i}=1 ; \mathrm{i}<\mathrm{N}-1 ; \mathrm{i}++)$

theta_itv10[(it+1)/itvl0][i] = theta[i];

for $(\mathrm{i}=0 ; \mathrm{i}<\mathrm{N} ; \mathrm{i}++)$

C_itvl0[(it+1)/itvl0][i] $=\mathrm{C}[\mathrm{i}]$;

$$
\}
$$

\} $\mathrm{t}=($ itend-1.0)*dt;

fprintf(fp_out, "DtC at it =\%d (\%12.6fln", itend-1, t); fprintf(fp_out, "i, x, DtCln");

for $(\mathrm{i}=0 ; \mathrm{i}<\mathrm{N} ; \mathrm{i}++)$

$\operatorname{DtC}[\mathrm{i}])$;

fprintf(fp_out, "\%d, \%12.6f, \%12.6fln", i, x[i],

fprintf(fp_out, "\n");

// theta

$\mathrm{t}=($ itend-1.0)*dt;

fprintf(fp_out, "theta at it =\%d (\%12.6fln", itend-1, t); fprintf(fp_out, "i, x, theta $\backslash$ n");

for $(\mathrm{i}=0 ; \mathrm{i}<\mathrm{N} ; \mathrm{i}++)$

fprintf(fp_out, "\%d, \%12.6f, \%12.6fln", i, x[i], theta[i]);

fprintf(fp_out, "\n");

// C

$\mathrm{t}=($ itend +0.0$) * \mathrm{dt}$;

fprintf(fp_out, "C at it $=\% d(t=\% 12.6 f) \backslash n "$, itend, $t)$; fprintf(fp_out, "i, x, C\n");

for $(\mathrm{i}=0 ; \mathrm{i}<\mathrm{N} ; \mathrm{i}++)$

fprintf(fp_out, "\%d, \%12.6f，\%12.6fln"，i，x[i],

$\mathrm{C}[\mathrm{i}])$;

fprintf(fp_out, "\n");

$/ / \mathrm{dC} / \mathrm{dt}$

fprintf(fp_out, "DtC $\backslash n ")$;

fprintf(fp_out, "i, x, ");

for $(\mathrm{j}=0 ; \mathrm{j}<\mathrm{itend} / \mathrm{itvl0} ; \mathrm{j}++)$

fprintf(fp_out, "t=\%12.6f, ", ((j+0.0)*itvl0)*dt);

fprintf(fp out, "\n");

for $(\mathrm{i}=0 ; \mathrm{i}<\mathrm{N}-1 ; \mathrm{i}++)\{$

fprintf(fp_out, "\%d, \%12.6f, ", i, x[i]);

for $(\mathrm{j}=0 ; \mathrm{j}<\mathrm{itend} / \mathrm{itvl0} ; \mathrm{j}++)$

fprintf(fp_out, "\%12.6f, ", DtC_itvl0[j][i]);

fprintf(fp_out, "\n");

\}

fprintf(fp_out, "\n");

// theta

fprintf(fp_out, "theta $\backslash n$ ");

fprintf(fp_out, "x, ");

for $(\mathrm{j}=0 ; \mathrm{j}<=$ itend $/ \mathrm{itv} 10 ; \mathrm{j}++)$

fprintf(fp_out, "t=\%12.6f, ", ((j+0.0)*itvl0)*dt);

fprintf(fp_out, "\n");

for $(\mathrm{i}=1 ; \mathrm{i}<\mathrm{N}-1 ; \mathrm{i}++)\{$

fprintf(fp_out, "\%12.6f, ", x[i]);

for $(\mathrm{j}=0 ; \mathrm{j}<=$ itend/itvl0; $\mathrm{j}++)$

fprintf(fp_out, "\%12.6f, ", theta_itv10[j][i]);

fprintf(fp_out, "\n");

\}

fprintf(fp_out, "\n");

// C

fprintf(fp_out, "Cln"); 


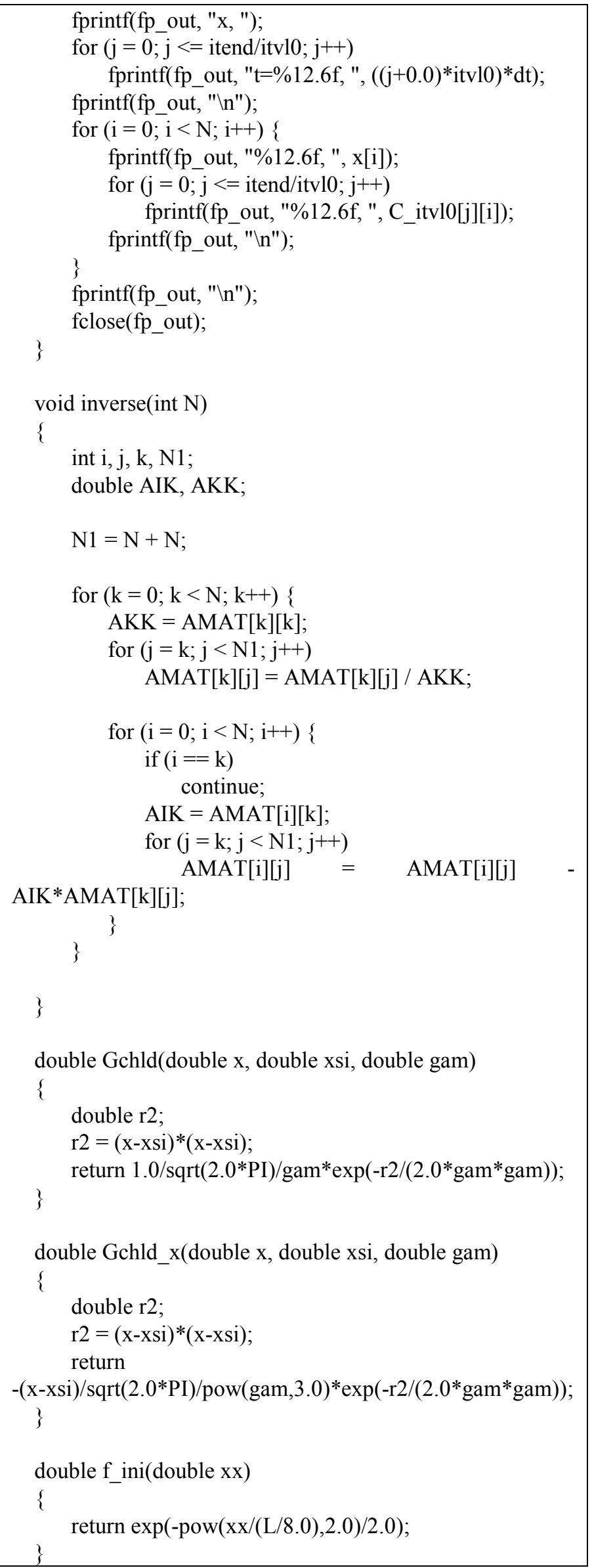

\section{B.3. FORTRAN code}

integer n, a(100), m, k

real*8 L, $\operatorname{tt}(100)$

real*8 $\mathrm{x}(401)$

real*8 $\mathrm{dx}$

real*8 $\mathrm{t}$

real*8 dt

integer it, itend, itvl, itv10

real*8 u

real*8 kpp

real*8 c(401)

real*8 dtc(401)

real*8 citvl0(1000,401), dtcitvl0(1000,401)

real*8 theta(401), qq(401)

real $* 8$ thetaitvl0 $(1000,401)$

real $* 8 \mathrm{c} 0, \mathrm{c} 1$

real*8 gam

real*8 alp

integer nitg, inino

integer $i, j, p$

real*8 amat $(4001,8001)$

real*8 vec0(2001)

real*8 vec1(2001)

real*8 tmp $(401)$

open(11,file='input 1.dat')

$\operatorname{open}(12$, file='calcu1.csv')

c

$\operatorname{read}(11, *) \mathrm{L}$

$\operatorname{read}(11, *) \mathrm{n}$

$\operatorname{read}(11, *) \mathrm{kpp}$

$\operatorname{read}(11, *) \mathrm{u}$

$\operatorname{read}(11, *) \mathrm{c} 0$

$\operatorname{read}(11, *) \mathrm{c} 1$

$\operatorname{read}(11, *) \mathrm{dt}$

$\operatorname{read}(11, *)$ itend

$\operatorname{read}(11, *)$ itvl

$\operatorname{read}\left(11,{ }^{*}\right)$ itvl0

$\operatorname{read}\left(11,{ }^{*}\right)$ gam

$\operatorname{read}(11, *)$ alp

$\operatorname{read}(11, *)$ nitg

c

$\mathrm{dx}=2.0 \mathrm{~d} 0 * \mathrm{~L} /(\mathrm{n}+0.0 \mathrm{~d} 0)$

c

$$
\begin{aligned}
& \text { do } 100 \mathrm{i}=1, \mathrm{n} \\
& \mathrm{x}(\mathrm{i})=-\mathrm{L}+0.5 \mathrm{~d} 0 * \mathrm{dx}+(\mathrm{i}-1.0+0.0 \mathrm{~d} 0) * \mathrm{dx} \\
& \mathrm{c}(\mathrm{i})=\operatorname{fini}(\mathrm{x}(\mathrm{i}), \mathrm{L})
\end{aligned}
$$

100 continue

c

$$
\begin{aligned}
& \text { do } 300 i=1, n \\
& \operatorname{do~} j=1, n \\
& \operatorname{amat}(i, j)=0.0 d 0 \\
& \operatorname{do} p=1, \operatorname{nitg} \\
& \quad \operatorname{amat}(i, j)=\operatorname{amat}(i, j)+\operatorname{Gchld}(x(j)-d x / 2.0 d 0
\end{aligned}
$$$$
1
$$

$+(\mathrm{p}-1.0+0.5) * \mathrm{dx} /(\operatorname{nitg}+0.0 \mathrm{~d} 0), \mathrm{x}(\mathrm{i}), \mathrm{gam}) * \mathrm{dx} /(\operatorname{nitg}+0.0 \mathrm{~d} 0)$ 

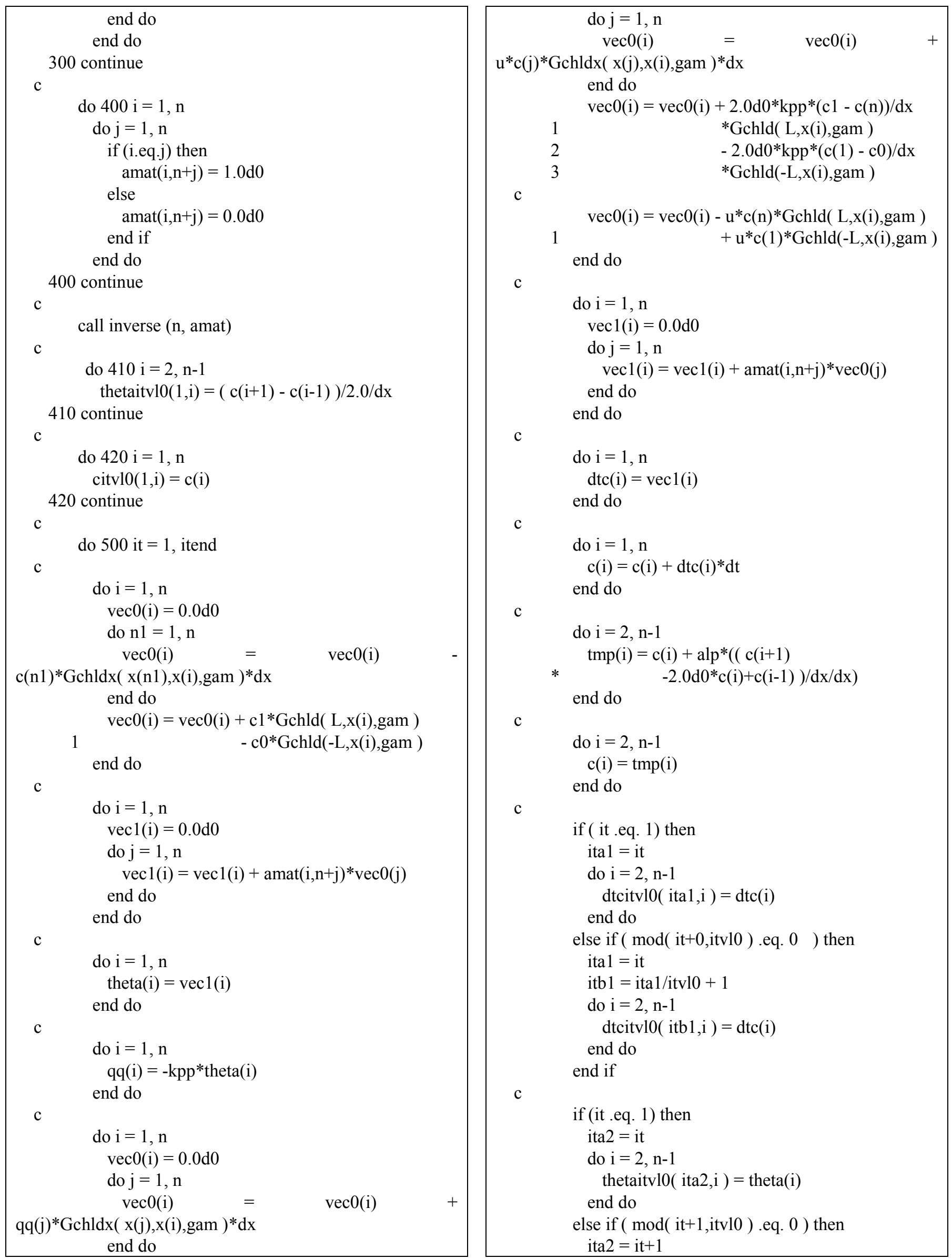

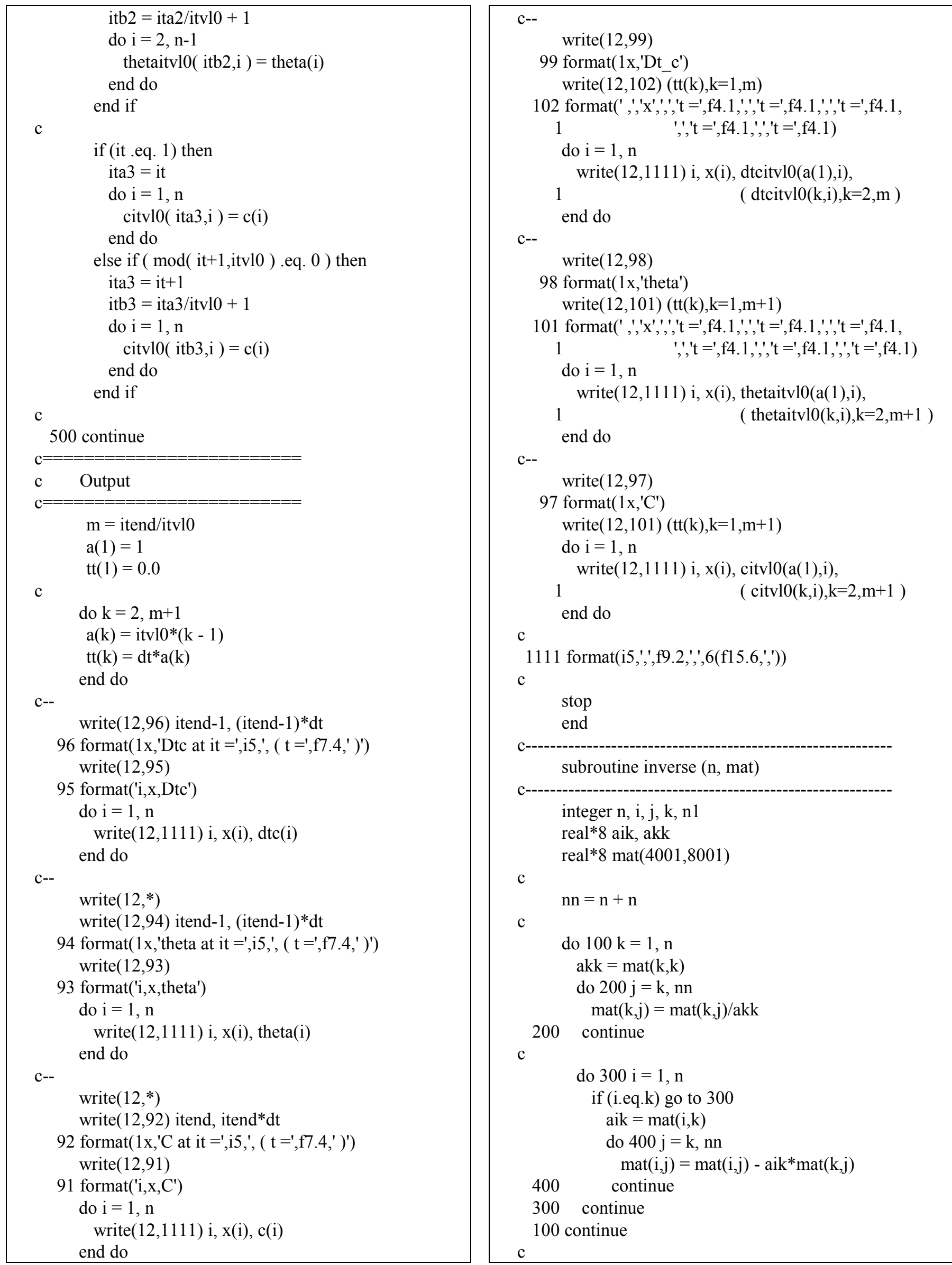


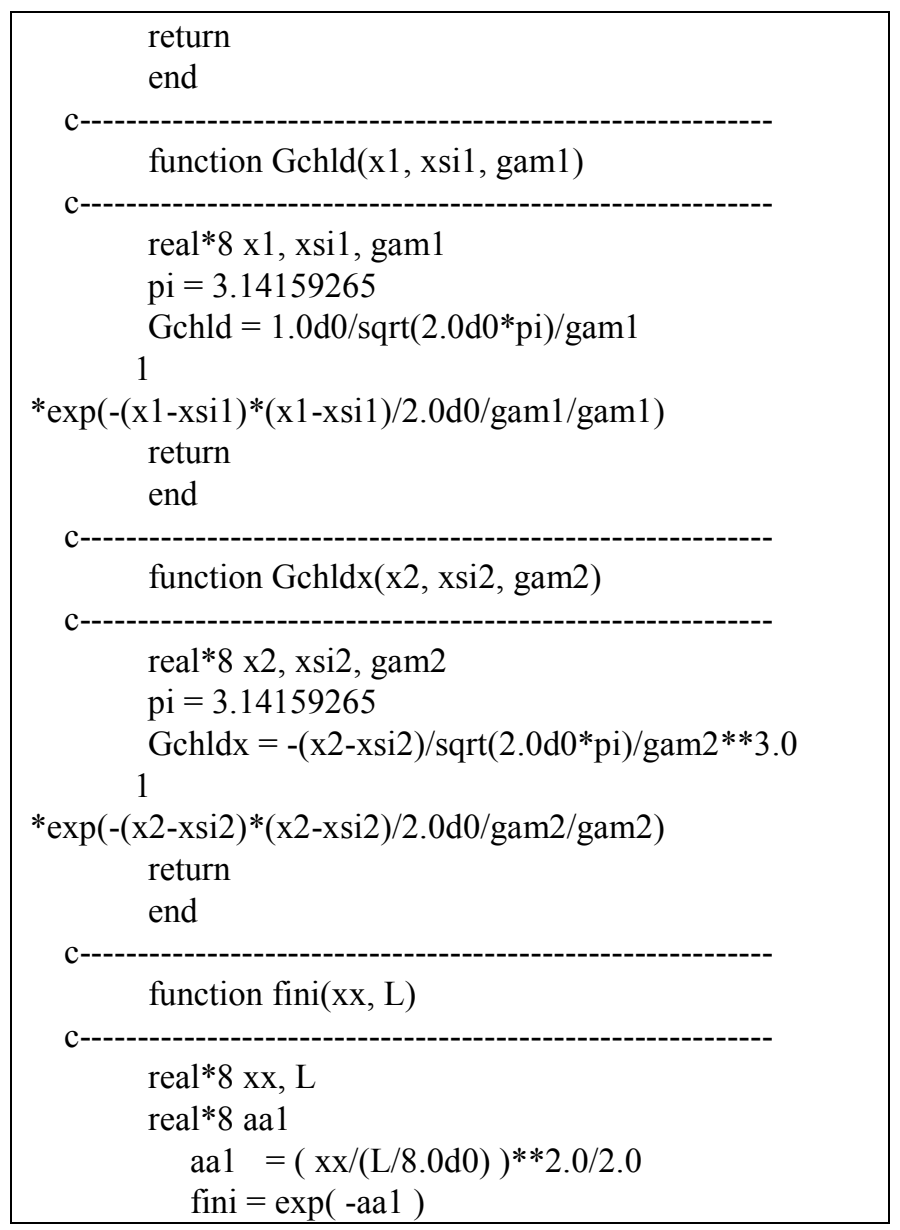

return

end

\section{References}

[1] H. Isshiki, "From Integral Representation Method (IRM) to Generalized Integral Representation Method (GIRM)," Applied and Computational Mathematics, Special Issue: Integral Representation Method and Its Generalization, (2015), under publication. http://www.sciencepublishinggroup.com/ journal/archive.aspx?journalid=147\&issueid $=-1$

[2] H. Isshiki, T. Takiya, and H. Niizato, "Application of Generalized Integral representation (GIRM) Method to Fluid Dynamic Motion of Gas or Particles in Cosmic Space Driven by Gravitational Force," Applied and Computational Mathematics, Special Issue: Integral Representation Method and Its Generalization, (2015), under publication. http://www.sciencepublishinggroup.com/journal/archive.aspx? journalid $=147 \&$ issueid $=-1$

[3] H. Isshiki, "Effects of Generalized Fundamental Solution (GFS) on Generalized Integral Representation Method (GIRM)," Applied and Computational Mathematics, Special Issue: Integral Representation Method and Its Generalization, (2015), under publication. http://www.sciencepublishinggro up .com/journal/archive. aspx?journalid=147\&issueid=-1

[4] H. Isshiki, "Application of the Generalized Integral Representation Method (GIRM) to Tidal Wave Propagation," Applied and Computational Mathematics, Special Issue: Integral Representation Method and Its Generalization, (2015), under publication. http://www.sciencepublishinggroup.com/ journal/archive.aspx?journalid=147\&issueid=-1 